\title{
Ernährung und Wachstum der Felchenpopulationen (Coregonus spp.) des Thuner- und Bielersees
}

\author{
Von Hans Rufli \\ Eidg. Technische Hochschule Zürich, Institut für Gewässerschutz und Wassertechnologie' ${ }^{1}$ )
}

Manuskript eingegangen am 8. März 1979

\begin{abstract}
Nutrition and growth of the coregonid fish populations in Lake Thun and Lake Biel The subject of this paper - nutrition and growth of Coregonus spp. in Lake Thun and Lake Biel became acute, when changes in their biology, as well as in the composition of the stocks were observed (due to eutrophication).

According to stomach analyses, Cladocera are the main food organisms of the populations in Lake Thun (local names: Kropfer, Albock, Brienzlig) and Lake Biel (Palée, Bondelle) during the period of growth (limited to May-September/October). In winter and spring they feed on Copepods and bottom organisms. The composition of the food differs little between populations (Palée prey on Bondelle-eggs during Bondelle spawning season, while Bondelle feed on Daphnia), except for the Kropfer, which ingests bottom organisms probably during the entire year.

Growth characteristics determined by scalimetry resulted in the following mean asymptotic length $\mathrm{L}_{\infty}$ and Ford's growth coefficient $\mathrm{k}$ : Albock: $\mathrm{L}_{\infty}=44 \mathrm{~cm}, \mathrm{k}=0.58 ;$ Brienzlig: $\mathrm{L}_{\infty}=36 \mathrm{~cm}, \mathrm{k}=0.55$; Bondelle: $\mathrm{L}_{\infty}=31 \mathrm{~cm}, \mathrm{k}=0.43$. In the past 25 years the Coregonus spp. in the two lakes experienced a considerable improvement in their growth (corresponds to Blaufelchen in Lake Constance prior to eutrophication, Albock has better growth). This improvement is mainly referred to better growth rates during the first two years of life. Later they lie below former values. The decrease, mainly of the stocks of Lake Biel, is probably caused by diseases and parasites.
\end{abstract}

\section{INHALTSVERZEICHNIS}

1. Einleitung und Problemstellung $\ldots \ldots \ldots \ldots \ldots \ldots \ldots \ldots \ldots \ldots \ldots \ldots \ldots \ldots \ldots$

2. Charakterisierung der Seen und ihrer Felchenpopulationen $\ldots \ldots \ldots \ldots \ldots \ldots \ldots \ldots \ldots$

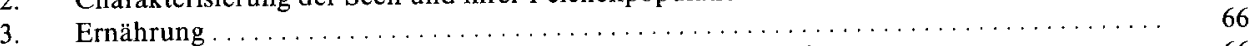

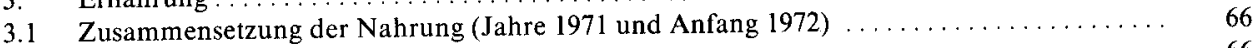

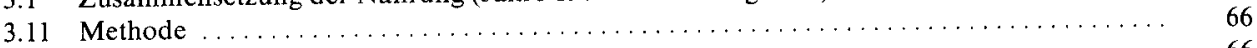

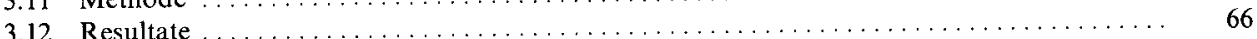

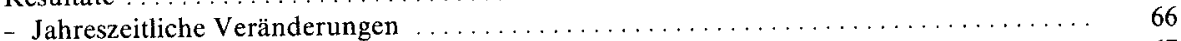

- Unterschiede in der Nahrung der einzelnen Formen . . . . . . . . . . . . . . . . . . . 67

- Beziehungen zwischen Mageninhalt und Zooplanktonpopulation im See . . . . . . . . 70

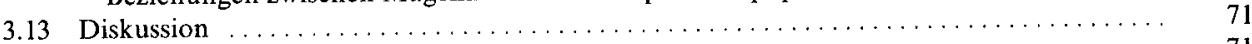

- Nahrung der Thuner- und Bielerseefelchen $\ldots \ldots \ldots \ldots \ldots \ldots \ldots \ldots \ldots \ldots \ldots \ldots \ldots$

1) Die Arbeit wurde an der Eidgenössischen Anstalt für Wasserversorgung, Abwasserreinigung und Gewässerschutz (EAWAG), Abteilung für Fischereiwissenschaften, ausgeführt. 


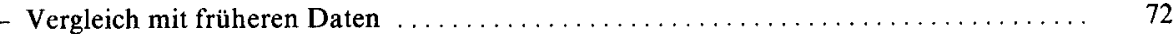

- Beziehung zwischen der Felchenernährung und der Zusammensetzung des Seeplanktons $\quad 73$

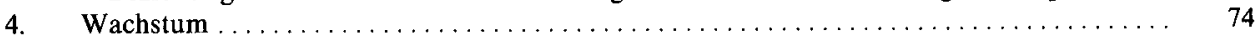

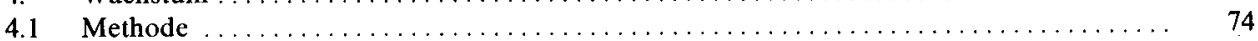

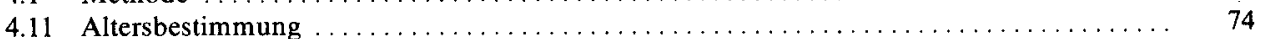

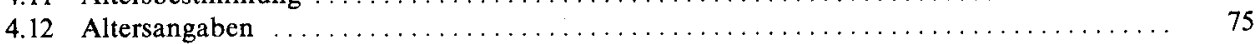

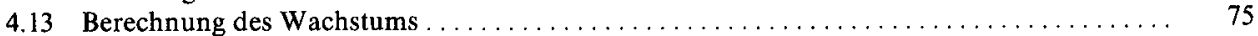

- Beziehung zwischen Schuppenlänge und Fischlänge .................... 75

- Rückberechnung der individuellen Längen und Berechnung

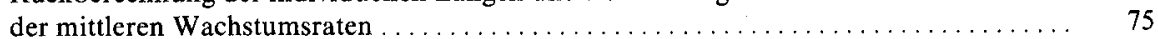

- Zuverlässigkeit der Ergebnisse . . . . . . . . . . . . . . . . . . . . . . . . . . . . 76

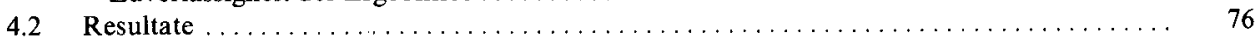

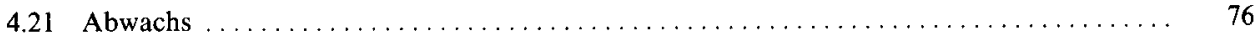

- Durchschnittswerte der Altersklassen . . . . . . . . . . . . . . . . . . . . . . . 76

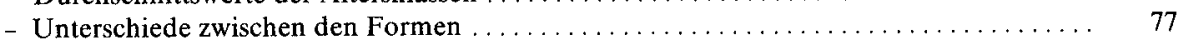

- Vergleich zwischen den Formen des Thüner- und Bielersees . . . . . . . . . . . . . 78

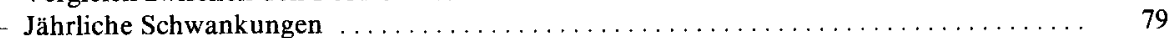

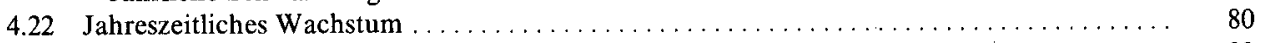

- Methode ................................................ 80

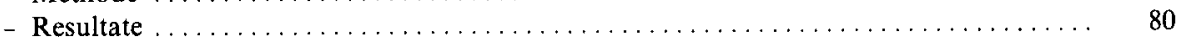

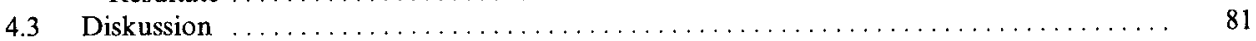

- Unterschiede zwischen den Formen des Thunersees ...................... 81

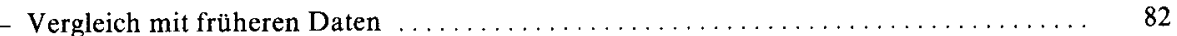

- Konsequenzen der Wachstumsveränderungen ......................... 86

- Vergleich des Wachstums der Thuner- und Bielerseefelchen

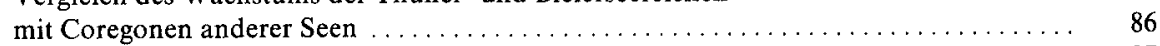

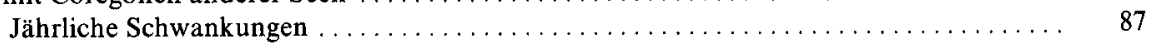

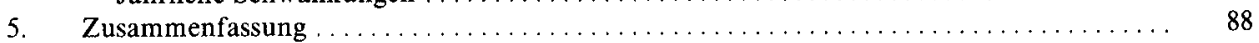

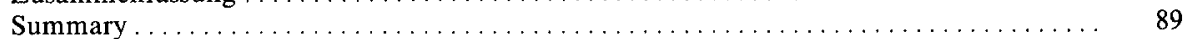

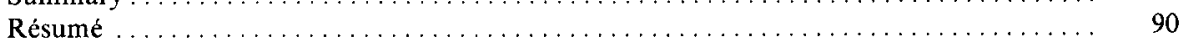

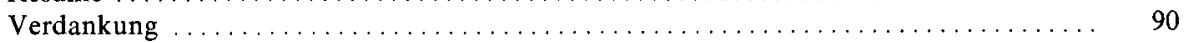

Literaturverzeichnis . . . . . . . . . . . . . . . . . . . . . . . . . . . . . . 91

\section{Einleitung und Problemstellung}

Als Folge von Umweltveränderungen haben sich Morphologie und Biologie der Coregonen und die arten- und rassenmässige Zusammensetzung der Felchenpopulationen im Thuner- und Bielersee in den letzten 25 Jahren verändert [47, 48]. Um eine Anpassung der Bewirtschaftung an die neuen Verhältnisse zu ermöglichen, ist die Kenntnis der gegenwärtigen Lebensgewohnheiten der Coregonen notwendig. Es ist Ziel dieser Arbeit, die Grundlagen dafür - Ernährung und Wachstum der Thuner- und Bielerseefelchen - zu liefern.

\section{Charakterisierung der Seen und ihrer Felchenpopulationen}

Eine Zusammenstellung der morphometrischen und hydrologischen Daten des Thuner- und Bielersees findet sich bei Nydegger [40] und bei Rufli [47, 48]. Der Thunersee ist ein tiefer, oligo-mesotropher Alpenrandsee (subtropisch). Beim Bielersee handelt es sich um einen flachen, eutrophen Mittellandsee (temperiert). Beide werden durch die Aare durchflossen, Wehre unterbinden allerdings eine Fischwanderung von See zu See. 
Wie aus den Untersuchungen von Rufli [47, 48] hervorgeht, lassen sich im Thunersee drei Felchenpopulationen, Kropfer, Albock und Brienzlig (Lokalnamen) ${ }^{2}$ ), unterscheiden $(5,28$ bzw. $78 \%$ des Gesamtbestandes). Im Bielersee sind zwei Formen, Palée und Bondelle, vorhanden (16 bzw. $84 \%$ des Bestandes). Diese Populationen sind charakterisiert durch ihre Kiemenreusenzahlen und weitere morphologische Merkmale, durch ihre Laichgewohnheiten [47], ihr Wachstum und zum Teil durch ihre Ernährung.

\section{Ernährung}

\subsection{Zusammensetzung der Nahrung (Jahre 1971 und Anfang 1972)}

\subsection{Methode}

Der Inhalt (zum Teil nur aliquote Teile) von 675 in Formol fixierten Magen wurde ausgezählt ${ }^{3}$ ). Der Darminhalt diente zu Kontrollzwecken. Aufgrund der Analyse von klar identifizierbaren Mageninhalten wird die durchschnittliche Nahrungszusammensetzung (in Prozenten) im Jahresverlauf für die einzelnen Felchenpopulationen dargestellt.

Zur Schätzung der Zooplankton-Zusammmensetzung im See standen (nach Möglichkeit monatliche) Planktonproben aus 0-15 m Tiefe im Thunersee und 0-20 $\mathrm{m}$ im Bielersee zur Verfügung. Die Resultate ergaben sich durch Auszählen von 4 bis 5 Teilproben, die aus dem gut durchmischten Planktonkonzentrat herauspipettiert wurden.

\subsection{Resultate}

\section{Jahreszeitliche Veränderungen}

Die Zusammensetzung der aufgenommenen Nahrung im Jahresverlauf ist in Abb. 1-4 dargestellt (bei weniger als drei Fischen sind die entsprechenden Felder leer gelassen).

Thunersee: Während der Wachstumsperiode von Mai bis September ernährt sich der Brienzlig hauptsächlich von Cladoceren, nämlich Daphnia, Bythotrephes und Leptodora. Im September kommen Chironomiden dazu. Im Herbst herrscht in der Brienzlignahrung noch weitgehend Daphnia vor, danach erscheinen Copepoden (im

2) Die Vielfalt der Schwierigkeiten in der Bestimmung der Felchenarten und-rassen führte zu unterschiedlichen wissenschaftlichen Bezeichnungen der Thuner- und Bielerseefelchen durch frühere Autoren $[15,57,69]$. Wir benützen deshalb in der vorliegenden Arbeit die oben erwähnten, lokal gebräuchlichen Namen. Unter dem Albock des Thunersees verstehen wir eine Form, welche höchstwahrscheinlich durch Hybridisierung der ursprünglichen Schläge Bodenalbock (Balchen) und Schwebalbock (Albock), wie sie Steinmann [57] beschrieb, entstand [47, 48].

3) Vom Februar 1971 bis März 1972 wurden im Thuner- und Bielersee monatlich 20-45 Fische, gesamthaft 872, für die vorliegende Arbeit gefangen. Weitere 1100 Felchen standen aus Fängen der Jahre 1969 und 1970 zur Verfügung. Die verwendeten Netze und Maschenweiten sind bei Rufli [47, 48] beschrieben. Das Originalmaterial befindet sich in der EAWAG/ETH, CH-6047 Kastanienbaum. Die Dissertation (Nr.5451) ist in der ETH Zürich erhältlich. 
Januar und Februar 1972 bildete Diaptomus den überwiegenden Anteil, im April 1971 Cyclops).

In der Zeit von Mai bis August (Wachstumsphase) besteht die Nahrung des Albocks ausschliesslich aus Daphnia und Bythotrephes, wobei Daphnia im Frühling, Bythotrephes im Sommer überwiegt. Wahrscheinlich spielt im September auch Leptodora eine wichtige Rolle (der Mageninhalt des einzigen im September gefangenen Albocks bestand zu $100 \%$ aus Leptodora). Während der übrigen Jahreszeiten dominiert Daphnia in der Albocknahrung. Im Oktober erscheinen auch Bythotrephes und Leptodora, im Januar vermutlich Chironomiden (nur zwei Alböcke gefangen).

Die wenigen Kropfer gestatten nur für die Monate Oktober und Februar eine Aussage. In beiden Monaten zeigten sämtliche Tiere eine einheitliche Nahrungszusammensetzung, mit einem überwiegenden Anteil an Chironomiden. Der Rest bestand aus unbestimmbaren, braunen, etwa 0,1-0,2 $\mathrm{mm}$ grossen Eiern und aus Daphnia.

Bielersee: Daphnia bildet den Hauptnahrungsorganismus der Bondelle. Im Frühling tragen auch Cyclops und Bosmina, im August vor allem Chironomiden und Cyclops zur Ernährung bei. Zahlenmässig ist Anfang Herbst Bosmina von Bedeutung. Im November beteiligen sich Leptodora und Copepoden an der Bondellenahrung. Nach der Laichzeit bis zu Beginn der Wachstumsperiode herrschen Bodennahrung (Pisidien, Tubificiden, Chironomus, Felcheneier) und Cyclops vor.

$\mathrm{Zu}$ Beginn der Wachstumsperiode im Mai überwiegt in der Palée-Nahrung Daphnia, daneben finden sich Cyclops und Bosmina. Beim einzigen im Juli gefangenen Palée bestand die Nahrung zu 93\% aus Daphnia. Gegen Ende der Wachstumsperiode (August, September) treten vorübergehend Chironomiden und Leptodora auf. Im Herbst und Winter dominiert erneut Daphnia, Anfang Herbst für kurze Zeit allerdings Cyclops. Während der Laichzeit tragen Bondelle-Eier wesentlich zur Palée-Ernährung bei.

Unterschiede in der Nahrung der einzelnen Formen

Thunersee: Die Nahrung des Kropfers (ausschliesslich Bodenorganismen) unterscheidet sich, soweit wir darüber Angaben besitzen, klar von derjenigen der übrigen Thunerseeformen. Weniger eindeutig differiert die Zusammensetzung der Mageninhalte zwischen Brienzlig und Albock (Abb. 1, 2). Schliesst man für den Vergleich die Monate mit weniger als vier Tieren aus (d.h. Januar, April, September, Oktober, Dezember), so sind die Unterschiede in der Nahrungszusammensetzung auffallend gering (Ausnahme: März). Innerhalb derselben Population kann sie allerdings stark variieren. Zum Teil kommen diese Differenzen durch unterschiedliche Ernährung verschiedener Grössenklassen derselben Form zustande, wie dies auch Elster [12] und Nilsson [35] feststellten. Beispielsweise fressen im Oktober alle Brienzlige mit Totallängen unter $30 \mathrm{~cm}$ Chironomiden, diejenigen über $30 \mathrm{~cm}$ hingegen Zooplankton. Von den letzteren ernähren sich wiederum die grössten Tiere mehr von Bythotrephes, die kleineren mehr von Copepoden. Soweit eine Gegenüberstellung gleicher Grössenklassen aufgrund einer genügenden Anzahl Tiere möglich ist, ergeben sich 
THUNERSEE: ALBOCK

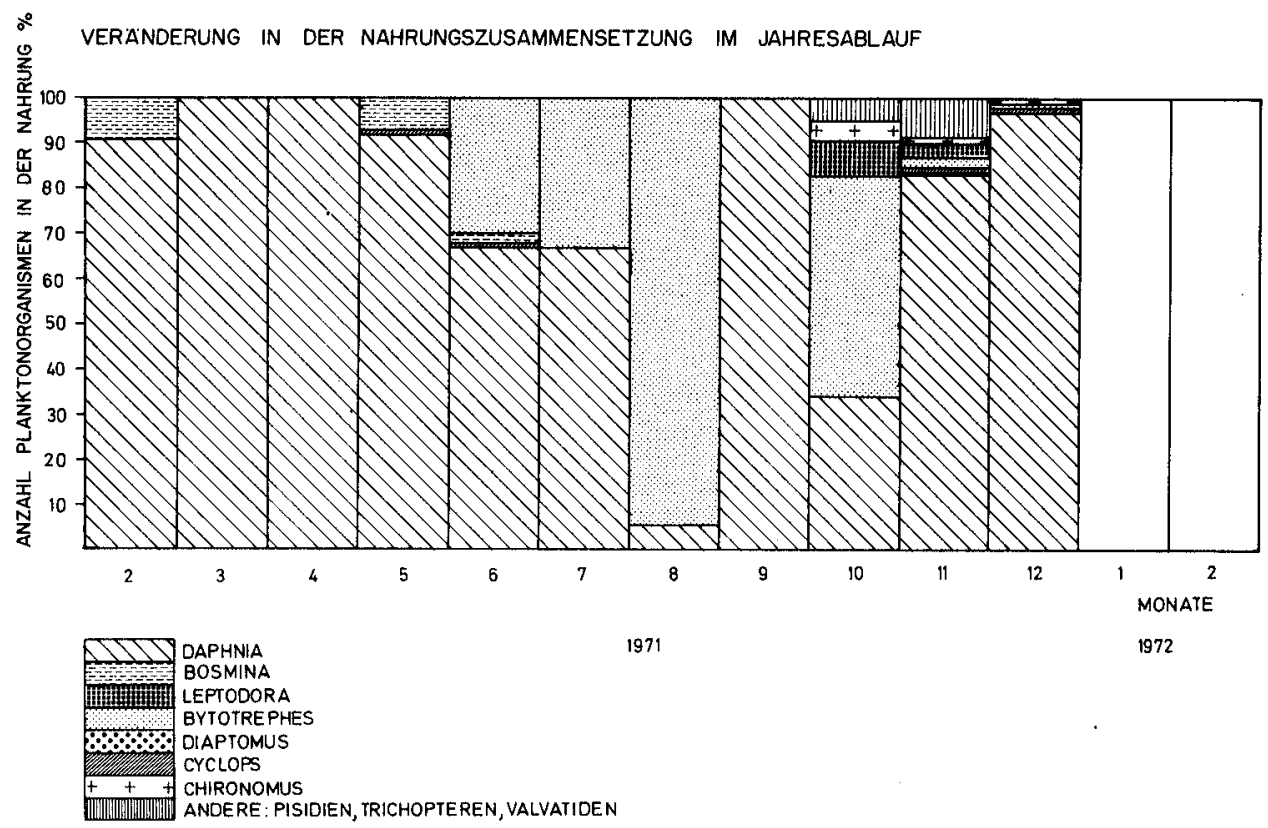

Abb. 1. Thunersee: Albock. Veränderung der Nahrungszusammensetzung im Jahresablauf 1971 und Anfang 1972.

Figure 1. Lake Thun: Albock. Annual changes in the composition of nutrition during 1971 and beginning of 1972 .

eindeutige Unterschiede einzig für den Monat März. Während dieser Zeit ernährt sich der Brienzlig stärker von Copepoden als der Albock. Diese Unterschiede bestehen vermutlich während des ganzen Winters (nur wenige Exemplare vorhanden).

Im allgemeinen differiert die Zusammensetzung der Nahrung zwischen Brienzlig und Albock nur wenig oder überhaupt nicht. Einzig beim Kropfer können deshalb Magenanalysen zur Identifizierung der Form beitragen.

Bielersee: Unterschiede in der Zusammensetzung der Nahrung zwischen Bondelle und Palée sind im Oktober, Dezember und Januar besonders ausgeprägt (Abb. 3, 4). Während der übrigen Monate differiert die Nahrungszusammensetzung relativ wenig. Berücksichtigt man in Anbetracht der Schwankungen innherhalb derselben Form nur Monatsmittelwerte aufgrund von mindestens fünf Tieren (d.h. Mai, November, Dezember), findet man nur noch im Dezember wesentliche Unterschiede zwischen den Formen. Eine genauere Analyse zeigt, dass die Differenzen während der Monate Mai, August, September und Oktober auf den Vergleich unterschiedlicher Grössenklassen zurückzuführen sind. Im Oktober fressen die grössten Bondelles und die kleinsten (in der Grösse aber entsprechenden) Palées 


\section{THUNERSEE: BRIENZLIG}
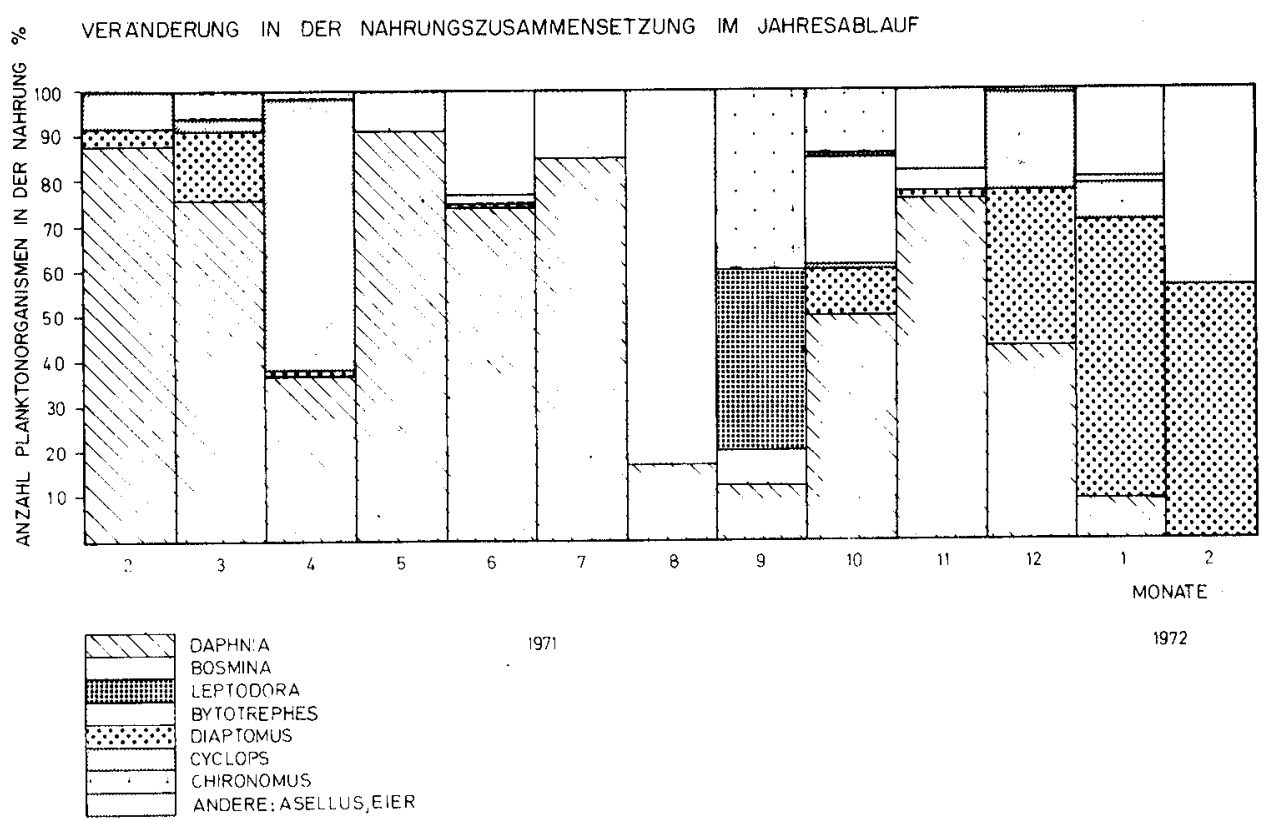

Abb. 2. Thunersee: Brienzlig. Veränderung der Nahrungszusammensetzung im Jahresablauf 1971 und Anfang 1972.

Figure 2. Lake Thun: Brienzlig. Annual changes in the composition of nutrition during 1971 and beginning of 1972 .

vorwiegend Cyclops. Die meisten der kleineren Bondelles ernähren sich aber vor allem von Daphnia und die kleinsten von Bosmina. In den Monaten Juli und November, in denen sich die untersuchten Fische in der Grösse entsprechen, sind keine Unterschiede bezüglich der Nahrungswahl zwischen den beiden Formen feststellbar. Tatsächliche Differenzen bestehen demnach einzig in den Monaten Dezember und wahrscheinlich Januar. Der bei der Palée feststellbare Frass von Bondelle-Eiern (Dezember) hängt nämlich nicht mit der Grösse zusammen, sondern mit der unterschiedlichen Laichzeit der beiden Formen. Laichreife Palées nehmen durchaus dieselbe Nahrung wie die Bondelles zu sich. Die Mehrheit der unreifen oder verlaichten Tiere ernährt sich jedoch ausschliesslich von Bondelle-Eiern (in einem der Magen befanden sich 900 Eier). Im Jahre 1971 konnten Felcheneier nur in den Magen verlaichter oder noch nicht geschlechtsreifer Tiere gefunden werden, während sich die laichreifen Fische vorwiegend von Plankton ernährten.

Zusammenfassend lässt sich festhalten, dass wesentliche Unterschiede in der Nahrungswahl zwischen Bondelle und Palée nur im Winter, vor allem während der Bondelle-Laichzeit, existieren. Der zu dieser Zeit ausschliesslich aus Bondelle-Eiern bestehende Mageninhalt der Palées gilt bei den Berufsfischern als ein Unterscheidungsmerkmal der Bielerseeformen. 


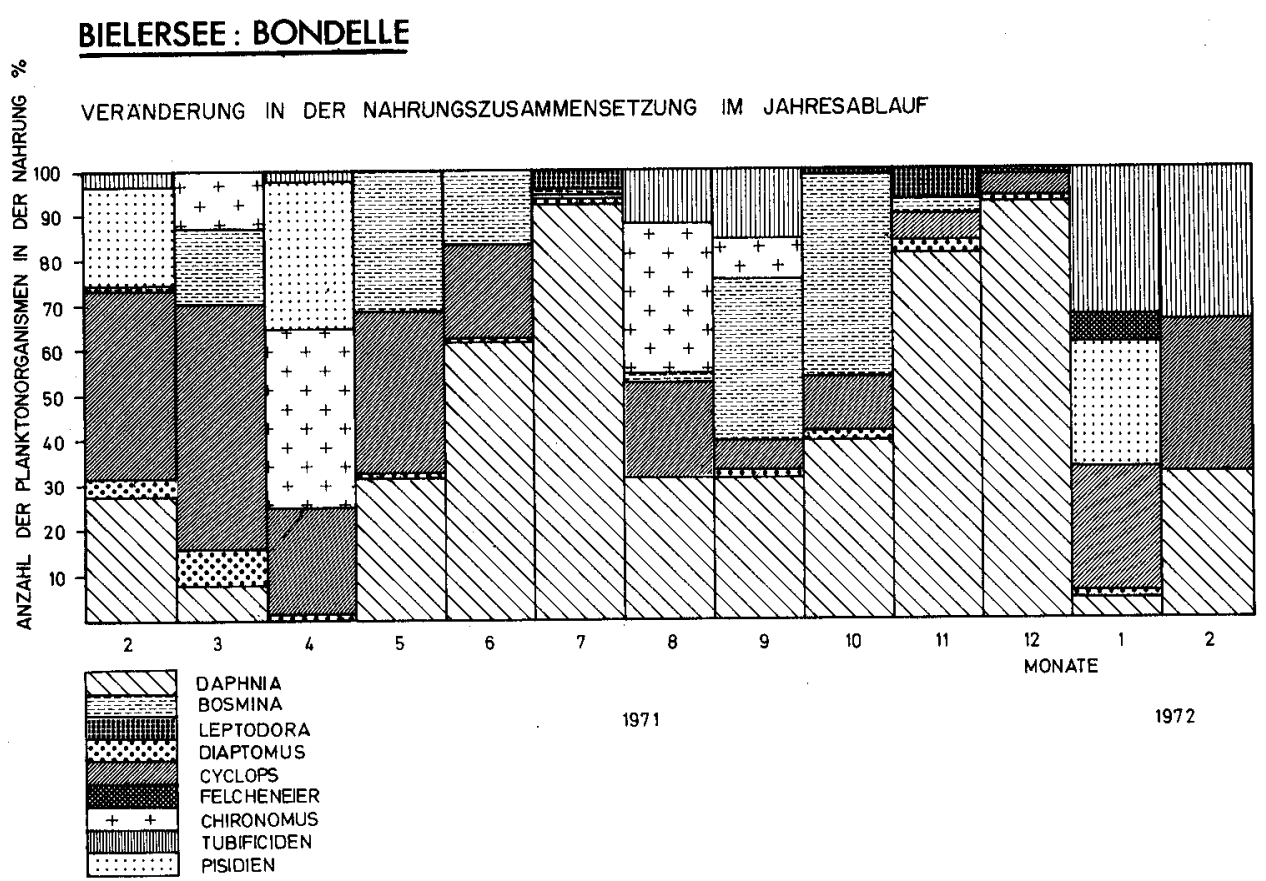

Abb.3. Bielersee: Bondelle. Veränderung der Nahrungszusammensetzung im Jahresablauf 1971 und Anfang 1972.

Figure 3. Lake Biel: Bondelle. Annual changes in the composition of nutrition during 1971 and beginning of 1972 .

Beziehungen zwischen Mageninhalt und Zooplanktonpopulation im See

Obwohl in der Zusammensetzung des Zooplanktons an verschiedenen Orten des Sees starke Schwankungen auftreten können [12], sind wir der Meinung, dass unsere Ergebnisse zumindest den groben Jahresverlauf der Verhältnisse in den obersten 0-20 $\mathrm{m}$ der beiden Seen erkennen lassen. Der Jahresverlauf des auf diese Weise erhaltenen Gehalts (in Prozenten) an einzelnen Planktern weist denn auch deutliche Parallelen zu jenem in andern, hinsichtlich Trophiezustand und morphologische Verhältnisse vergleichbaren Seen auf $[33,66]$.

Thunersee: Ein Vergleich der Zusammensetzung des Seeplanktons mit der aufgenommenen Felchennahrung zeigt (Abb. 1, 2, 5), dass die Cladoceren Daphnia, Bythotrephes und Leptodora von Albock und Brienzlig bevorzugt gefressen werden (prozentualer Gehalt in der Nahrung höher als im Seeplankton). Für den Brienzlig gilt dies auch in bezug auf Cyclops. Bosmina und Diaptomus hingegen, sowie Cyclops beim Albock, treten in der Nahrung nur beschränkt auf (prozentualer Gehalt in der Nahrung kleiner als im Seeplankton). 


\section{BIELERSEE: PALÉE}

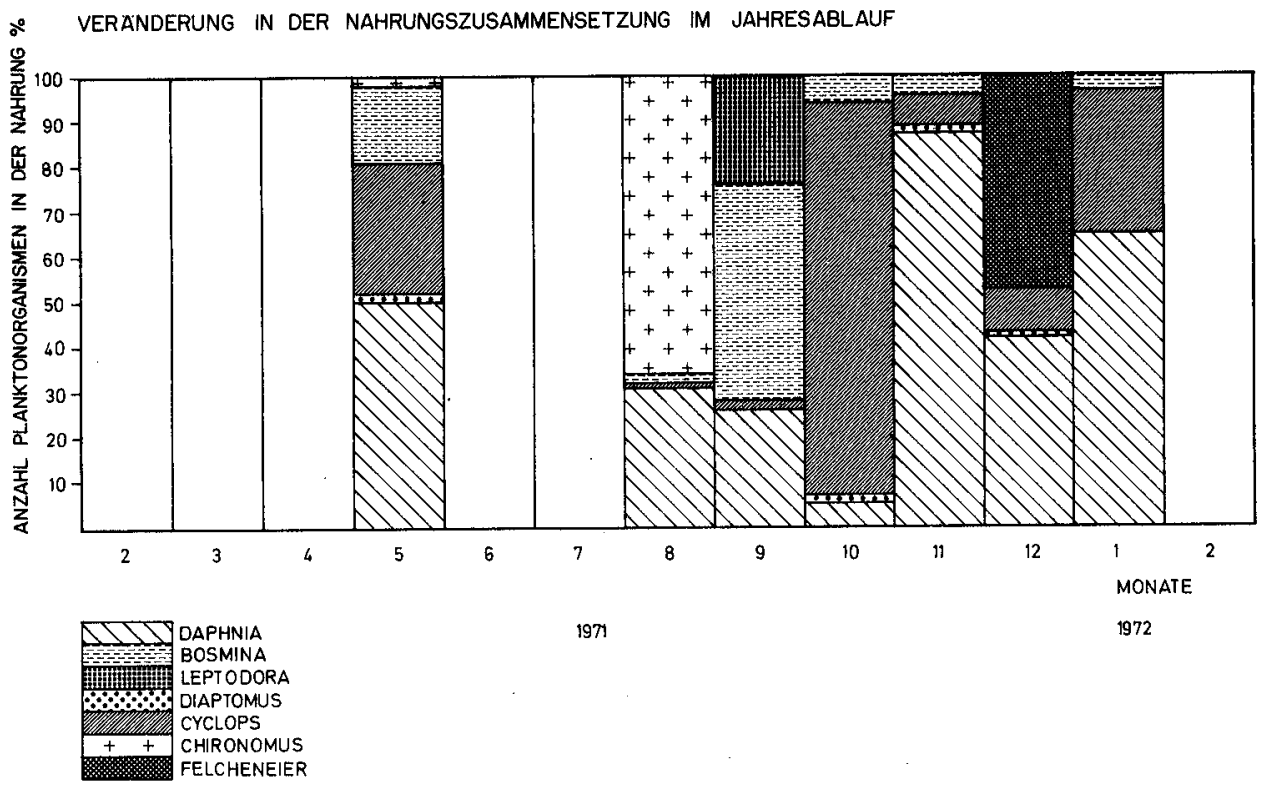

Abb. 4. Bielersee: Palée. Veränderung der Nahrungszusammensetzung im Jahresablauf 1971 und Anfang 1972.

Figure 4. Lake Biel: Palée. Annual changes in the composition of nutrition during 1971 and beginning of 1972.

Bielersee: Daphnia und Leptodora (letztere vor allem von grossen Felchen), in geringem Masse auch Bosmina und Cyclops, sind die bevorzugten Nahrungsorganismen der Bielerseefelchen (zum Teil grössere Konzentration im Magen als im See oder gleiche; Abb.3, 4, 6). In der Coregonennahrung wenig vertreten ist dagegen Diaptomus. Diaphanosoma wird überhaupt nicht gefressen.

\subsection{Diskussion}

Nahrung der Thuner- und Bielerseefelchen

Aufgrund der Nahrungsgewohnheiten handelt es sich bei den Thuner- und Bielerseefelchen vornehmlich um Schwebfelchen, nur der Kropfer ist als Bodenfelchen anzusehen [27, 57, 71]. Die Zusammensetzung der Nahrung dieser Schwebfelchen stimmt gut mit jener der Blaufelchen des Bodensees [2, 12], des Schluchsees [28], der Felchen südalpiner Seen $[4,65]$ und einiger Formen britischer Seen überein $[20,54$, 55, 64]. Charakteristisch für die jährliche Wachstumsperiode (Mai bis August, September) ist der hohe Anteil an Cladoceren (Daphnia und Bythotrephes im Thunersee, Daphnia und Bosmina im Bielersee). Gelegentlich treten unter den 
THUNERSEE

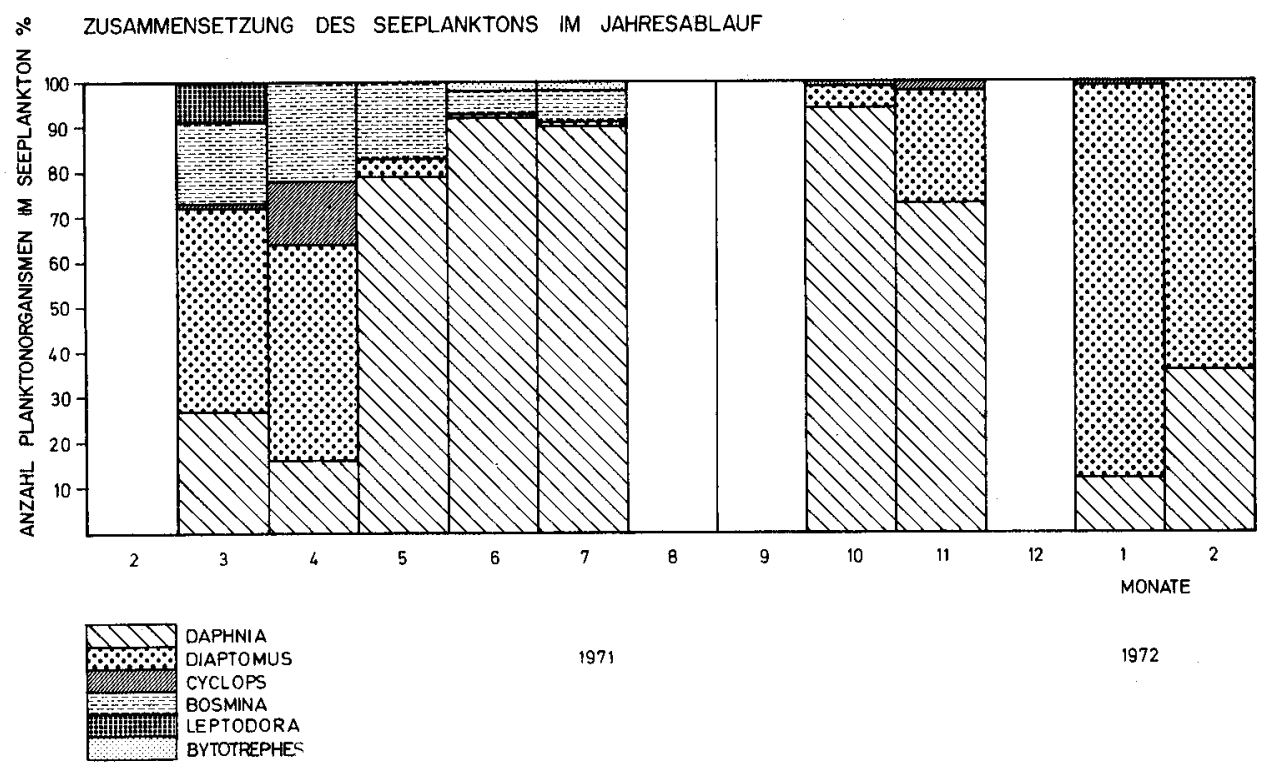

Abb. 5. Thunersee. Zusammensetzung des Seeplanktons im Jahresablauf.

Figure 5. Lake Thun. Composition of the plankton during 1971 and beginning of 1972 .

Nahrungskomponenten auch Chironomidenlarven und -puppen, Cyclops, Diaptomus, Tubificiden oder Flugnahrung auf [12, 35, 57, 70]. Jungfische [24, 27, 35, 39, 57] wurden in keinem der untersuchten Magen festgestellt.

Die Winternahrung unterscheidet sich von derjenigen des Sommers durch das vermehrte Auftreten von Copepoden und Bodenorganismen, hauptsächlich Chironomiden. Von Bedeutung sind auch Pisidien, Valvatiden, Eurycercus, Asellus, Tubificiden und Eier $[4,17,36,69,72]$ verschiedenster Herkunft. Frass von Felcheneiern durch fremde Coregonenpopulationen (Palée) wurde auch von andern Autoren beobachtet [14, 20]; Steinmann [57] erwähnt dieses Phänomen selbst beim Kropfer.

\section{Vergleich mit früheren Daten}

Verglichen mit den Ergebnissen der Steinmannschen Untersuchungen aus den Jahren 1944 und 1950 [56, 57], hat sich die Ernährung der Thunerseefelchen, trotz den bedeutenden quantitativen und qualitativen Veränderungen des Nahrungsangebots (Eutrophierung), nicht wesentlich verändert. Während beispielsweise die dem Kropfer entsprechende Form des Bodensees (Kilch) von der Zunahme des Planktons profitiert und sich heute im Pelagial in wenigen Metern Tiefe aufhält [22], ernährt sich der Kropfer des Thunersees wie 1944 von Bodennahrung. 


\section{BIELERSEE}
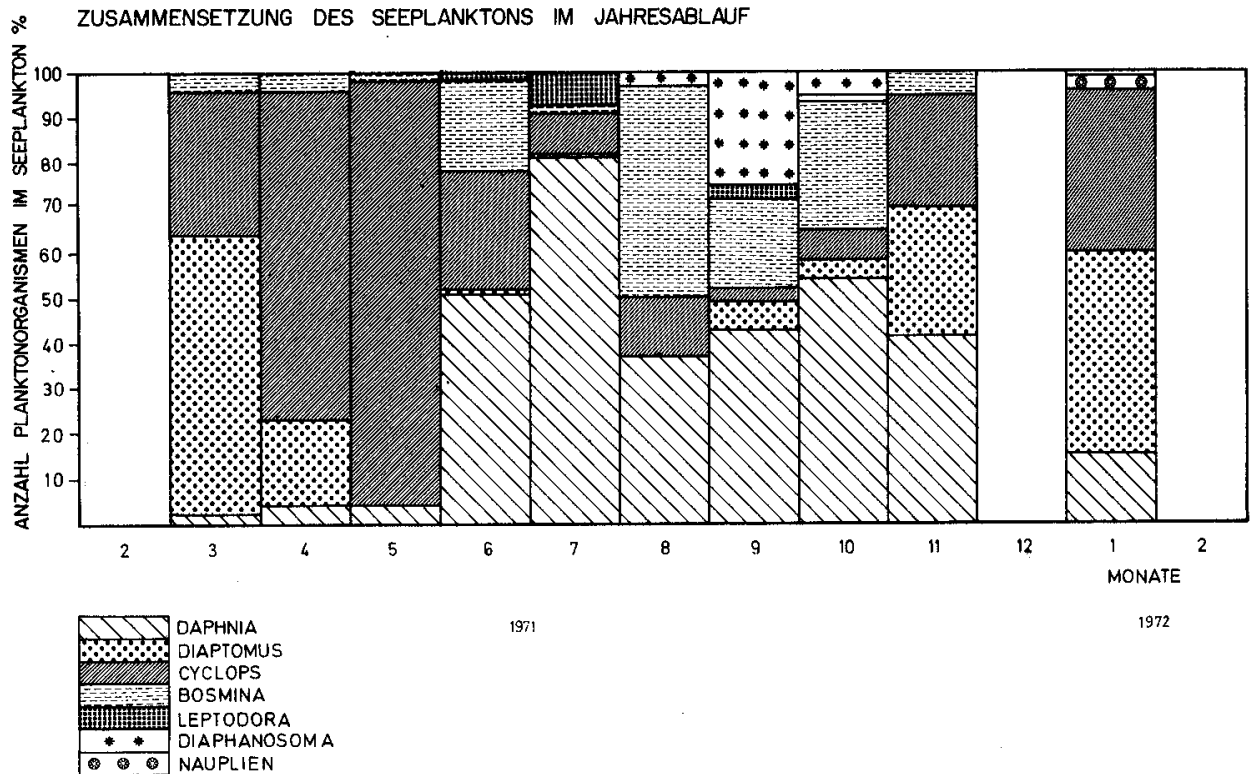

197

1972

Abb.6. Bielersee. Zusammensetzung des Seeplanktons im Jahresablauf.

Figure 6. Lake Biel. Composition of the plankton during 1971 and beginning of 1972.

Im Gegensatz zu damals finden sich in der Nahrung des Brienzligs heute in grösseren Mengen Bodenorganismen. Es ist nicht ausgeschlossen, dass sich diese Umstellung auf das Wachstum positiv ausgewirkt hat [62].

Für den heutigen Albock ist ein Vergleich mit den damaligen Boden- und Schwebalböcken (Hybridisierung [47, 48] schwieriger. Es scheint jedoch, dass die heutige Form in bezug auf die Ernährung eher dem früheren Schwebalbock entspricht.

Beziehung zwischen der Felchenernährung und der Zusammensetzung des Seeplanktons

Differenzen in der Zusammensetzung der Felchennahrung und jener des Seeplanktons lassen sich bis zu einem gewissen Grad durch die unterschiedliche Vertikalverteilung von Felchen und Planktonorganismen in der Zeit der stabilen Schichtung erklären [2, 11]. Obwohl sich der Aujenthaltsort der Felchen stark nach der Tiefenverteilung der Hauptnährtiere richtet [13], sind die Coregonen als stenotherme Tiere an einen bestimmten Temperaturbereich gebunden [67]. Diaphanosoma fehlt in der Felchennahrung vermutlich deshalb, weil die Felchen den Bereich der warmen Oberflächenschichtung meiden, in der sich die vor allem im Sommer und Herbst auftretende Diaphanosoma befindet $[42,51]$. Anders verhält sich Daphnia (grösste 
Dichten in den obersten $20 \mathrm{~m}$ ) $[12,33,42,53]$, die bis in das Aufenthaltsgebiet der Felchen reicht.

Die relativ starken Schwankungen im Gehalt an Bosmina (besonders deutlich bei den Bielerseefelchen) sind wahrscheinlich die Folge von Strömungen und andern hydrographischen Einflüssen, denen Bosmina stärker unterworfen ist als Daphnia. Dies führt zu oft unregelmässigen und stark wechselnden Tiefenverbreitungskurven dieser Planktonart [12].

Die festgestellte Vorliebe und Spezialisierung der Felchen auf wenige oder bei gewissen Tieren auf irgendeine der offerierten Planktonarten [28, 57, 70] lässt vermuten, dass die Coregonen fähig sind, aktiv eine gewisse Auswahl innerhalb der angebotenen Nahrung zu treffen. Nach Galbraith [18] (Untersuchungen an Regenbogenforellen und Yellow Perch) muss, neben einer Abhängigkeit der aufgenommenen Menge an grossen Nahrungsorganismen von der relativen Grösse der Kiemenreusen-Zwischenräume [12, 13, 24], eine weitere Form der Nahrungswahl existieren. Vermutlich wird die Nahrungsauslese durch die schwarmweise Verteilung der Planktonorganismen $[50,66]$ erleichtert, indem die Felchen die von den gewünschten Organismen bevorzugten Räume aufsuchen [4].

\section{Wachstum}

\subsection{Methode}

Für die Untersuchungen standen 872 Fische aus Fängen der Jahre 1971 und 1972 sowie 1100 aus den Jahren 1969 und 1970 aus dem Thuner- und Bielersee zur Verfügung (vgl. Fussnote 3).

\subsection{Altersbestimmung}

Zur Bestimmung des Alters wurde die bei Coregonen klassische Methode der Scalimetrie angewendet. Die benützten lateralen Schuppen (Schuppenreihe unmittelbar unter der Seitenlinie, auf der Höhe des vorderen Randes der Bauchflosse) weisen gegenüber ventralen eine wesentlich geringere Streuung auf, sowohl zwischen Schuppen des gleichen Tieres als auch zwischen solchen verschiedener Tiere derselben Länge [4].

Als Kennzeichen eines Annulus wurden die bei Berg und Grimaldi [5] (S.229-230) definierten Kriterien verwendet, davon hauptsächlich die Erscheinung des «cutting over».

Nach Möglichkeit wurden an mindestens drei Schuppen die Annuli bestimmt und ihre Abstände vom Zentrum in oraler Richtung in der Vergrösserung ausgemessen (Projektionsmikroskop Projectina AG, Vergrösserung 40,4). Als Schuppenlänge $l_{1}$, $1_{2}, 1_{3}, \ldots 1$ (nach 1, 2, 3 Jahren ... bzw. beim Fang) wurden die Mittelwerte der Daten aller ausgemessenen Schuppen eines Tieres betrachtet. Obwohl Kontrollbestimmungen der Annuli bei künstlich aufgezogenen Bielerseefelchen bekannten Alters (maximal $4^{+}$) keinerlei Schwierigkeiten bereiteten, liess sich das Alter bei Felchen aus dem See nicht immer eindeutig bestimmen. Eine Häufigkeitsverteilung des jährlichen und jahreszeitlichen Zuwachses (rückberechnet nach Lea) pro Alter und 
Jahresklasse (Tiere gleichen Geburtsjahres) liess jedoch zweifelhafte Altersbestimmungen mit vermutlich falschem oder eventuell fehlendem Annulus erkennen und korrigieren $[11,28]$.

\subsection{Altersangaben}

Da kein einheitliches Datum existiert, das dem Schlüpfen der Brut aller Formen nur einigermassen entspricht, wird der 1.Januar als Geburtstag aller Coregonenformen gewählt.

Die Bezeichnung des Alters erfolgt in der Form $0^{+}, 1^{+}, 2^{+}$und in Altersklassen 0, 1, 2. Unter $0^{+}$Tieren bzw. Fischen der Altersklasse 0 verstehen wir solche, die im ersten Lebensjahr, d.h. in der Zeit zwischen ihrer Geburt und dem 1. Januar des Jahres, in dem sie ihren ersten Annulus bilden, gefangen werden.

\subsection{Berechnung des Wachstums}

Beziehung zwischen Schuppenlänge und Fischlänge

Das Fehlen einer genügenden Menge kleiner Fische in unseren Daten würde eine für kleine Fischlängen $\mathbf{L}$ unzutreffende Kurve ergeben. Da bei künstlich aufgezogenen Bondelles die Schuppenbildung bei einer Grösse von 2 bis $4 \mathrm{~cm}$ einsetzt (dieser Bereich deckt sich mit den Werten anderer Coregonen [19, 21], wurde die Fischlänge $\mathrm{L}=2,5 \mathrm{~cm}$ mit der Schuppenlänge $\mathrm{l}=0$ ebenfalls zur Berechnung der Kurve herangezogen (gleiches Gewicht wie die Gesamtheit der restlichen Punkte).

Aus den Resultaten der nach der Methode der kleinsten Quadrate berechneten Regressionskurven der verschiedenen Formen:

$\begin{array}{ll}\text { Kropfer } & 1(\times 40,4)=-8,254+0,325 \mathrm{~L}+0,00029 \mathrm{~L}^{2} \\ \text { Albock } & 1(\times 40,4)=-6,371+0,240 \mathrm{~L}+0,00061 \mathrm{~L}^{2} \\ \text { Brienzlig } & 1(\times 40,4)=-8,914+0,355 \mathrm{~L}+0,00009 \mathrm{~L}^{2} \\ \text { Palée } & 1(\times 40,4)=-7,766+0,302 \mathrm{~L}+0,00042 \mathrm{~L}^{2} \\ \text { Bondelle } & 1(\times 40,4)=-9,165+0,362 \mathrm{~L}+0,00024 \mathrm{~L}^{2}\end{array}$

geht hervor, dass

a) die Kurven die Bezeichnung 1/L auch für kleine Fischlängen in zufriedenstellender Weise beschreiben;

b) die relative Grösse der Schuppen mit wachsender Fischlänge leicht zunimmt;

c) die Kurven der verschiedenen Formen sich sehr ähnlich sehen [4, 10].

Vergleicht man die Kurven der Thuner- und Bielerseefelchen, lassen sich wesentliche Unterschiede nur zwischen den grösseren Exemplaren des Brienzligs und des Albocks aus dem Thunersee feststellen (praktisch dieselben Unterschiede weisen Brienzlig und Albock des Brienzersees auf).

Rückberechnung der individuellen Längen und Berechnung der mittleren Wachstumsraten

Die Rückberechnung der individuellen Längen $L_{1}, L_{2}, L_{3}, \ldots L_{n}$ nach $1,2,3, \ldots N$ 
vollendeten Lebensjahren erfolgte auf der Basis der berechneten Eichkurve $1=a+b L+c^{2}$. Individuelle Abweichungen von der Beziehung $1 / L$ wurden durch Anwendung des von Segerstrale [52] vorgeschlagenen Korrekturverfahrens berücksichtigt.

Die statistischen Werte (Mittel, Standardabweichung der Normalverteilungen) des jährlichen und jahreszeitlichen Zuwachses wurden, nach Alter und Jahresklassen getrennt, von den individuellen Werten berechnet. Die Bestimmung der mittleren Jahresendlängen erfolgte unter Berücksichtigung sämtlicher Einzelwerte. Für die Berechnung der mittleren asymptotischen Länge $\mathrm{L}_{\infty}$ und von Fords Wachstumskoeffizient $\mathrm{k}$ der Ford-Walford-Beziehung [43] wurden Jahresendlängen benützt, die sich aus dem Mittel der Zuwachsraten der einzelnen Jahrgänge (mit mehr als 10 Individuen) ergaben.

\section{Zuverlässigkeit der Ergebnisse}

Nichtrepräsentativer Fang, selektive Mortalität (z.B. durch Vorwegfang der Vorwüchser) oder ein falsches Schuppenlänge-Fischlänge-Verhältnis als Rückberechnungsgrundlage können zu Differenzen der rückberechneten Jahresendgrössen $L_{1}$, $\mathrm{L}_{2}$ von Fischen unterschiedlichen Alters führen (Rosa Lees Phänomen [29]).

Wie aus einem Vergleich des Wachstums (d.h. der rückberechneten Längen) der einzelnen Jahrgänge (Altersklassen) hervorgeht,

a) treten in unseren Ergebnissen, soweit sie auf mehr als 10 Individuen beruhen, solche Unterschiede in der Grösse von $\mathrm{L}_{1}, \mathrm{~L}_{2}$ nicht auf;

b) sind bei allen Altersklassen die Zuwachsraten während eines einzelnen Jahres einheitlich gut oder schlecht;

c) entwickeln sich gewisse Brienzlige im dritten oder vierten Lebensjahr zu Voroder Langsamwüchsern, obwohl ihr Wachstum während der ersten Jahre durchschnittlich war (deshalb keine Differenzen unter a).

Beim Brienzlig ergibt dies bei der Berechnung der mittleren Jahresendlängen zu kleine Werte im vierten und fünften Altersjahr infolge selektiver Mortalität.

Die Wachstumskurven des Kropfers und des Palées wurden anhand von insgesamt nur 18 bzw. 51 Tieren berechnet und sind mit entsprechender Vorsicht aufzunehmen. Da die Bestände beider Populationen in den letzten 25 Jahren sehr abgenommen haben, werden die Resultate trotzdem dargestellt.

Bei den übrigen Formen erachten wir die Ergebnisse aus obigen Gründen als im Sinne von Lees Phänomen unverfälscht und die Fänge als repräsentativ.

\subsection{Resultate}

\subsection{Abwachs}

Durchschnittswerte der Altersklassen

Thunersee: Zwischen den Formen des Thunersees bestehen deutliche Unterschiede im Wachstumsverlauf (Abb.7): Albock: $\mathrm{L}_{\infty}=44 \mathrm{~cm}, \mathrm{k}=0,58$; Brienzlig: $\mathrm{L}_{\infty}=$ $36 \mathrm{~cm}, \mathrm{k}=0,55$. 


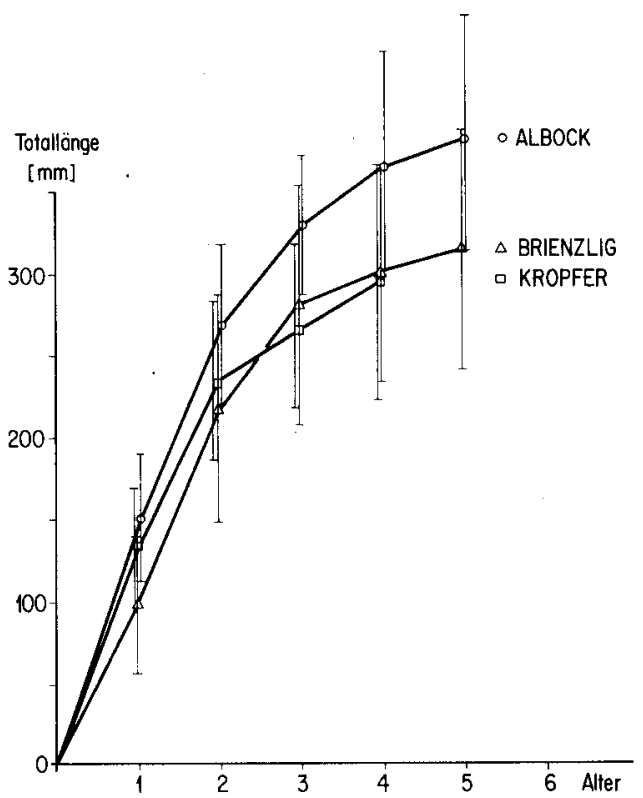

Abb. 7. Mittlere Jahresendlängen der Thunerseefelchen mit 95\%-Vertrauensgrenzen. Figure 7. Mean lengths of Coregonus spp. of Lake Thun with 95\% confidence limits.

Das Wachstum des Albocks ist durch einen durchwegs guten Jahreszuwachs gekennzeichnet. Besonders auffallend ist die Zunahme während der ersten beiden Jahre.

Die Kurve des Brienzligs zeigt im ersten Lebensjahr einen überraschend geringen Zuwachs. Erst im zweiten Lebensjahr erfolgt die grösste Längenzunahme, während sie in der Folge wieder deutlich kleiner wird (vgl. oben).

Der Kropfer besitzt im ersten Lebensjahr einen starken Jahreszuwachs, der in den folgenden Jahren kontinuierlich abnimmt.

Bielersee: Die Kurven der Bondelle und Palée sehen sich sehr ähnlich (Abb.8): Bondelle: $\mathrm{L}_{\infty}=31 \mathrm{~cm}, \mathrm{k}=0,43$. Auffallend ist ein sehr guter Zuwachs in den ersten beiden Lebensjahren. Vom dritten Jahr an ist die Längenzunahme wesentlich kleiner.

\section{Unterschiede zwischen den Formen}

Thunersee: Der Brienzlig besitzt gegenüber dem Albock bereits am Ende des ersten Lebensjahres signifikant ( $p<0,001)$ kleinere Längen (Abb.7). Bei einer Totallänge des Brienzligs von $10 \mathrm{~cm}$ beträgt die Differenz $5 \mathrm{~cm}$. Dieser Unterschied vergrössert sich im vierten und fünften Lebensjahr auf durchschnittlich $6,6 \mathrm{~cm}$ (vgl. S. 83). 
Das Wachstum des Kropfers differiert nur während der ersten beiden Lebensjahre von dem des Brienzligs $(p<0,001$ am Ende des ersten, $p<0,05$ am Ende des zweiten Jahres). Nach den mittleren Jahreslängen ist der Kropfer am Ende des ersten Jahres um durchschnittlich 3,7 cm (Totallänge 13,5 cm), am Ende des zweiten Jahres um $1,8 \mathrm{~cm}$ grösser (Totallänge $23,5 \mathrm{~cm}$ ). Durch den verhältnismässig guten Zuwachs des Brienzligs im dritten Jahr sind die Längen des Kropfers in der Folge im Mittel sogar etwas kleiner.

Bielersee: Trotz dem ähnlichen Verlauf der Wachstumskurven der beiden Bielerseefelchen sind die mittleren Jahresendlängen der Palées bereits vom ersten Jahr her grösser $(p<0,001)$ als diejenigen der Bondelles (Abb. 8).

Die anfängliche Längendifferenz von $1 \mathrm{~cm}$ (Totallänge $17,5 \mathrm{~cm}$ ) nach einem $\mathrm{Jahr}$ vergrössert sich dabei auf durchschnittlich $2 \mathrm{~cm}$ nach dem vierten Lebensjahr (Totallänge $31,4 \mathrm{~cm}$ ).

MITTLERE JAHRESLÄNGE DER BIELERSEEFELCHEN

MIT 95\% VERTRAUENSGRENZEN

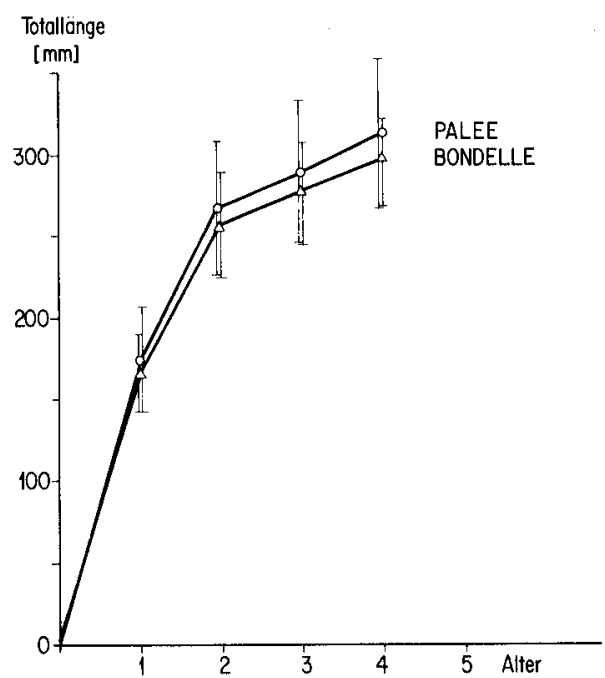

Abb. 8. Mittlere Jahresendlängen der Bielerseefelchen mit 95\%-Vertrauensgrenzen. Figure 8 . Mean lengths of Coregonus spp. of Lake Biel with $95 \%$ confidence limits.

Vergleich zwischen den Formen des Thuner- und Bielersees

Die Felchen des Bielersees übertreffen in ihrem Wachstum während des ersten Lebensjahres die schnellstwachsende Thunerseeform, den Albock, um 2,4 cm 
(Palée) und 1,5 cm (Bondelle) $(\mathrm{p}<0,001)$. Nach dem zweiten Altersjahr nähern sich die Bielerseefelchen (Abflachung der Wachstumskurve) den bis anhin langsamer wachsenden Kropfern und Brienzligen $(p<0,001)$. Die mittlere Jahresendlänge der Palées ist deshalb nach dem vollendeten vierten Lebensjahr zwar noch signifikant grösser als jene des Brienzligs und des Kropfers $(p<0,05)$, die Differenz beträgt aber nach anfänglich $7,7 \mathrm{~cm}$ und $4,0 \mathrm{~cm}$ nur noch $1,3 \mathrm{~cm}$ und $1,9 \mathrm{~cm}$. Die mittlere Länge der Bondelle unterscheidet sich von jener des Brienzligs nach vollendetem dritten Lebensjahr und nach einer maximalen Differenz von $6,8 \mathrm{~cm}$ überhaupt nicht mehr $(\mathrm{p}<0,05)$.

\section{Jährliche Schwankungen}

Thunersee: Beim Albock war der Zuwachs im Jahre 1971 bei den untersuchten Jahresklassen 1968 bis 1970 signifikant grösser als in den Vergleichsjahren 1970 $(p<0,001)$ und $1969(p<0,01)$. Für den Zuwachs im zweiten Lebensjahr ergaben sich Z. B. Differenzen von 3,3 cm zu 1970 und $1,0 \mathrm{~cm}$ zu 1969 (Tab. 1). Besseren Zuwachs ergab auch das Jahr 1969 gegenüber $1970(p<0,01)$ und $1968(p<0,05)$. Das Wachstum in den Jahren 1970 und 1968 unterscheidet sich nicht.

Nach der Stärke des Zuwachses lassen sich die Jahre folgendermassen einordnen: $1971>1969>1970=1968$.

Tabelle 1. Jahreszuwachs der Jahre 1968-1971 beim Albock.

Table 1. Albock. Annual length increments of the years 1968-1971.

\begin{tabular}{|c|c|c|c|c|c|c|c|c|c|}
\hline \multirow[t]{2}{*}{ Jahr } & \multicolumn{3}{|c|}{ Zuwachs im 1.Lebensjahr } & \multicolumn{3}{|c|}{ Zuwachs im 2.Lebensjahr } & \multicolumn{3}{|c|}{ Zuwachs im 3. Lebensjahr } \\
\hline & Mittel & $\begin{array}{l}\text { Standard- } \\
\text { abweichung }\end{array}$ & $\mathrm{N}$ & Mittel & $\begin{array}{l}\text { Standard- } \\
\text { abweichung }\end{array}$ & $\mathbf{N}$ & Mittel & $\begin{array}{l}\text { Standard- } \\
\text { abweichung }\end{array}$ & $\mathrm{N}$ \\
\hline 1968 & 14,8 & 1,8 & 32 & & & & & & \\
\hline 1969 & 15,8 & 2,1 & 37 & 12,2 & 1,9 & 31 & & & \\
\hline 1970 & 14,7 & 0,9 & 15 & 10,9 & 1,7 & 37 & 5,5 & 1,2 & 31 \\
\hline 1971 & & & & 13,2 & 1,3 & 15 & 8,0 & 0,9 & 18 \\
\hline
\end{tabular}

Beim Brienzlig ergab das Jahr 1971 bei den Jahrgängen 1967 bis 1970 einen hochsignifikant besseren Zuwachs $(\mathrm{p}<0,001)$ als 1969 und 1970 (Tab.2). Die Differenzen betrugen im dritten Lebensjahr 2,2 cm zu 1970 und $1,9 \mathrm{~cm}$ zu 1969.

Ordnet man diese Jahre nach der Stärke der Zuwachsraten, ergibt sich folgendes Bild: $1971>1969=1970$.

Tabelle 2. Jahreszuwachs der Jahre 1969-1971 beim Brienzlig.

Table 2. Brienzlig. Annual length increments of the years 1969-1971.

\begin{tabular}{|c|c|c|c|c|c|c|}
\hline \multirow[t]{2}{*}{$\mathrm{Jahr}$} & \multicolumn{3}{|c|}{ Zuwachs im 3. Lebensjahr } & \multicolumn{3}{|c|}{ Zuwachs im 4. Lebensjahr } \\
\hline & Mittel & Standardabweichung & $\mathbf{N}$ & Mittel & Standardabweichung & $\mathrm{N}$ \\
\hline 1969 & 6,4 & 2,1 & 43 & 2,7 & 0,9 & 27 \\
\hline 1970 & 6,1 & 1,6 & 56 & 2,4 & 0,8 & 56 \\
\hline 1971 & 8,3 & 2,1 & 48 & 4,1 & 1,6 & 29 \\
\hline
\end{tabular}

Bielersee: Die Bondelles des Jahrgangs 1968 weisen im Jahre 1971 einen besseren Abwachs auf als $1970(<0,001)$ (Tab.3). Der Zuwachs im vierten Lebensjahr war 
im Jahre 1971 um $0,9 \mathrm{~cm}$ grösser, also um mehr als einen Drittel. Ebenso wuchsen die Felchen im Jahre 1969 besser als $1970(\mathrm{p}<0,001)$, mit Unterschieden von $1 \mathrm{~cm}$ im zweiten und 1,4 cm im dritten Lebensjahr. Die Jahre 1968 und 1970 ergaben dagegen keine statistisch gesicherten Abweichungen im Wachstum $(p<0,05)$. Für einen Vergleich der Verhältnisse in den Jahren 1969 und 1971 fehlen die Zuwachswerte gleicher Altersklassen. Die Jahre lassen sich nach der Grösse des Längenwachstums folgendermassen anordnen:

$1971<1970=1968$.
$1969>$

Tabelle 3. Jahreszuwachs der Jahre 1968-1971 bei der Bondelle.

Table 3. Bondelle. Annual length increments of the years 1968-1971.

\begin{tabular}{|c|c|c|c|c|c|c|c|c|}
\hline \multirow[t]{2}{*}{ Jahr } & \multicolumn{3}{|c|}{ Zuwachs im 2. Lebensjahr } & \multicolumn{3}{|c|}{ Zuwachs im 3. Lebensjahr } & \multicolumn{2}{|c|}{ Zuwachs im 4. Lebensjahr } \\
\hline & Mittel & $\begin{array}{l}\text { Standard- } \\
\text { abweichung }\end{array}$ & $\mathrm{N}$ & Mittel & $\begin{array}{l}\text { Standard- } \\
\text { abweichung }\end{array}$ & $\mathrm{N}$ & Mittel & $\begin{array}{l}\text { Standard- } \\
\text { abweichung }\end{array}$ \\
\hline 1968 & 8,5 & 1,0 & 11 & & & & & \\
\hline 1969 & 9,0 & 0,9 & 207 & 3,3 & 1,3 & 11 & & \\
\hline 1970 & 8,0 & 1,9 & 9 & 1,9 & 0,6 & 207 & 1,4 & 0,7 \\
\hline 1971 & & & & & & & 2,2 & 0,6 \\
\hline
\end{tabular}

\subsection{Jahreszeitliches Wachstum}

\section{Methode}

Der jahreszeitliche Zuwachs wurde am gleichen Fisch, als Differenz zwischen der Länge während der letzten Annulusbildung und der Länge beim Fang, berechnet. Obwohl eine schwache Beziehung zwischen dem jahreszeitlichen Zuwachs und der Länge nach einem Jahr besteht, führt diese Art der Berechnung, soweit die monatlichen Mittelwerte auf mehr als zehn Beobachtungen beruhen, zu nur geringen Fehlern [4].

\section{Resultate}

Thuner- und Bielersee: Der Verlauf des jahreszeitlichen Wachstums für Albock, Brienzlig und Bondelle ist nach Altersklassen getrennt in den Abb.9-11 aufgetragen. Die Daten des Kropfers und der Palée sind zu spärlich, als dass man den jahreszeitlichen Zuwachs verfolgen könnte.

a) Nach den graphischen Darstellungen erstreckt sich die Wachstumsperiode der Thuner- und Bielerseefeichen von Mai bis Oktober. Der Zeitpunkt des Wachstumsstopps dürfte für ältere Fische $\left(3^{+}\right)$Ende September, für jüngere Ende Oktober eintreten.

b) Nach einem allmählichen Beginn des Wachstums Anfang Mai folgt von Mitte Mai bis August, bei den jüngeren Fischen bis Mitte September, eine Periode starken Zuwachses. Bis zur vollständigen Stagnation nimmt das Wachstum in der Folge kontinuierlich ab. 

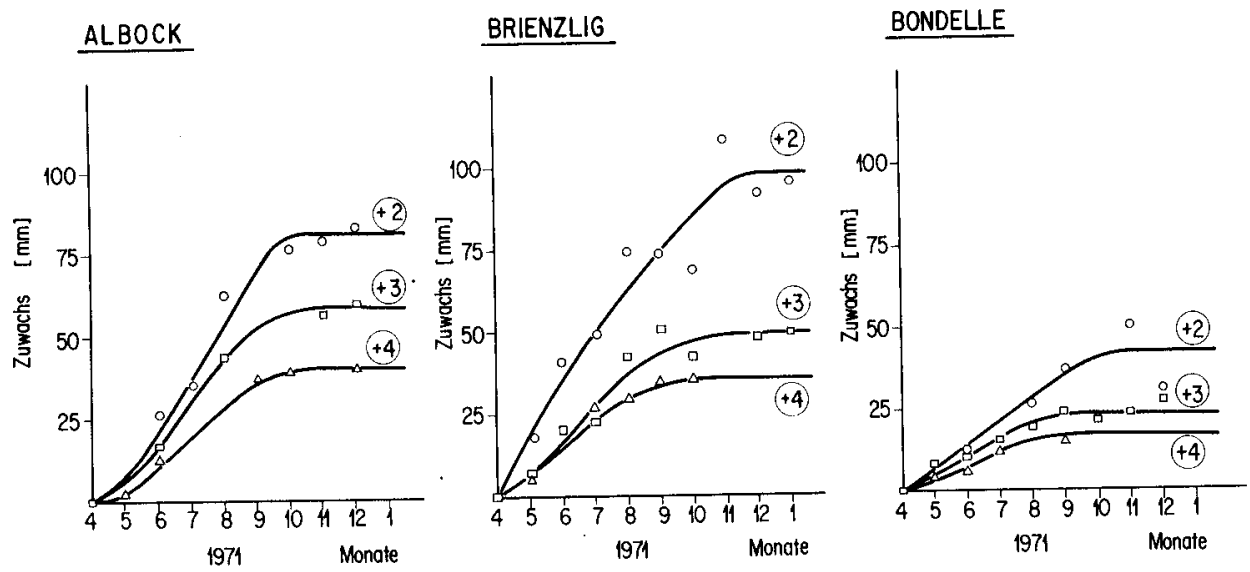

Abb.9. Verlauf des jahreszeitlichen Wachstums beim Albock des Thunersees, getrennt nach Altersklassen. (In den Monaten Februar und März erfolgt kein Zuwachs.)

Figure 9. Seasonal growth of the Albock of Lake Thun according to age classes. (There is no increase during February and March.)

Abb. 10. Verlauf des jahreszeitlichen Wachstums beim Brienzlig des Thunersees, getrennt nach Altersklassen. (In den Monaten Februar und März erfolgt kein Zuwachs.)

Figure 10. Seasonal growth of the Brienzlig of Lake Thun according to age classes. (There is no increase during February and March.)

Abb. 11. Verlauf des jahreszeitlichen Wachstums bei der Bondelle des Bielersees, getrennt nach Altersklassen. (In den Monaten Februar und März erfolgt kein Zuwachs.)

Figure 11. Seasonal growth of the Bondelle of Lake Biel according to age classes. (There is no increase during February and March.)

c) Das jahreszeitliche Wachstum ist in den ersten zwei bis drei Lebensjahren besonders ausgeprägt. Der durchschnittliche Zuwachs pro Monat beträgt während der Zeit des stärksten Wachstums:

\begin{tabular}{lll}
\hline & $1^{+}$ & $2^{+}$ \\
\hline Albock & $2,1 \mathrm{~cm}$ & $1,4 \mathrm{~cm}$ \\
Brienzlig & $1,8 \mathrm{~cm}$ & $0,9 \mathrm{~cm}$ \\
Bondelle & - & $0,3 \mathrm{~cm}$ \\
\hline
\end{tabular}

\subsection{Diskussion}

\section{Unterschiede zwischen den Formen des Thunersees}

Steinmann fasste Brienzlig und Kropfer unter dem Begriff «Kleinfelchen» zusammen. Trotz dem stark verbesserten Wachstum hat dieser Ausdruck noch immer seine Berechtigung. Beide Formen verfügen nämlich über denselben, im Vergleich zum Albock geringen Abwachs. Signifikante Unterschiede zwischen Brienzlig und Kropfer bestehen nur am Ende des ersten und schwach signifikant am Ende des 
zweiten Altersjahres. Sie entstehen durch einen unerwartet geringen Zuwachs des Brienzligs, der durch den stärkeren Zuwachs im zweiten Jahr zu einem Knick in der Wachstumskurve führt (Abb. 7).

Die ausgedehnte Laichzeit des Brienzligs und Kropfers hat zur Folge, dass die Schlüpfdaten von Angehörigen derselben Form bis zu sechs Monaten auseinanderliegen können. Da die Nachkommen der Sommerlaicher (Schlüpfzeit: Oktober, November) ihren ersten Annulus erst im zweiten Winter, gleichzeitig mit den Winterlaichern, bilden, sind erstere bis zu diesem Zeitpunkt maximal sechs Monate älter. Sie sollten dementsprechend länger sein. Im Vergleich zum winterlaichenden Albock erwartet man deshalb von Brienzlig und Kropfer einen relativ hohen Zuwachs im ersten Jahr. Beim Brienzlig trifft das Gegenteil zu.

Die Ursache dieser überraschenden Feststellung könnte trotzdem mit der früheren Laichzeit zusammenhängen. Die Nachkommen sommerlaichender Tiere schlüpfen zu einer Zeit, in der die Produktion an Plankton zurückgeht. Die Brut des Brienzligs, die gegenüber der Albockbrut ohnehin weniger Nahrungsreserven in Form des Dotters besitzt [47], hat zudem eine längere Phase der Nahrungsknappheit durchzustehen. Beim Albock findet die kritische Zeitspanne des Übergangs zur planktischen Ernährung später, etwa in der Zeit der steigenden Planktonproduktion, statt (laichreife Alböcke werden bis Anfang März gefangen). Es wäre deshalb denkbar, dass die stärkere Beanspruchung der Reserven beim Brienzlig während seines ersten Winters eine geringere Längenzunahme in der folgenden Wachstumsperiode bewirkt. Vergleicht man den ersten Jahreszuwachs sommerlaichender und winterlaichender Brienzlige, ergeben sich bei ersteren tatsächlich signifikant kleinere $\mathrm{Zu}$ wachsraten $(\mathrm{p}<0,05)$. Man könnte daher vermuten, dass zwischen dieser Benachteiligung der Sommerlaicher und der allgemeinen Tendenz der Laichzeitverschiebung in den Dezember und noch später [47] ein Zusammenhang besteht. Entsprechende Veränderungen der Laichzeiten haben bei allen Formen des Thunersees sowie beim Brienzlig des Brienzersees stattgefunden und sind wohl noch immer im Gange [47].

Der gute Zuwachs beim Kropfer (zum Teil ebenfalls Sommerlaicher) lässt sich allerdings nicht auf dieselbe Weise erklären. Die 18 Exemplare dieser Form, deren Laichzeit nur bei 7 Tieren bekannt ist, gestatten aber keine genauere Analyse. Die Ursache des unterschiedlichen Verhaltens von Brienzlig und Kropfer, wie z.B. Wüchsigkeit, Nahrung, Feinde oder andere Faktoren, bleibt daher unbekannt.

\section{Vergleich mit früheren Daten}

Olofsson [41], Runnström [49] und Svärdson [58, 59] konnten durch den Besatz von coregonenfreien Seen mit verzwergten Felchen («transplantations») zeigen, dass das Wachstum durch Umwelteinflüsse drastisch verändert werden kann. (Diese Feststellung widerspricht den Theorien Waglers [70], wonach der Abwachs wohl das spezifischste Merkmal einer Art darstellt.) Es lässt sich daraus schliessen, dass ähnliche Wachstumsverbesserungen auch innerhalb desselben Sees vor sich gehen können, wenn die nötigen Umweltveränderungen eintreten sollten. Beispiele dafür sind die Felchenarten des Bodensees [16, 22, 36], deren Wachstum als Folge der Eutrophierung stark zugenommen hat. 
Ein entsprechender Vergleich der heutigen Wachstumskurven der Thunerseefelchen mit den Angaben von Steinmann $[56,57]$ ergibt denn auch eine wesentliche Verbesserung des Abwachses bei allen Formen (Abb.12). Am auffallendsten sind die Veränderungen beim Brienzlig. Steinmann bezeichnete ihn infolge seines schwachen Wuchses als Zwergalbock. Diese Form besitzt heute mit zwei Jahren dieselbe Länge, die sie früher nach Vollendung des dritten Lebensjahres erreicht hatte $(22 \mathrm{~cm})$. Nach drei Jahren ist der heutige Brienzlig mit $28 \mathrm{~cm}$ durchschnittlich $7 \mathrm{~cm}$ länger! Es ist deshalb nicht erstaunlich, dass die Fischer diese Form nicht mehr als Brienzlig erkennen, um so mehr als parallel dazu Änderungen in der Laichzeit und Morphologie dieses Fisches einhergingen [47, 48].

Wie aus den Untersuchungen von Rufli [47, 48] hervorgeht, handelt es sich beim heutigen Albock höchstwahrscheinlich um eine Mischform der beiden Steinmannschen Grossfelchen Schweb- und Bodenalbock. Da sich diese Schläge in ihrem Wachstum kaum unterschieden, dürfte die Vermischung allein keine Änderung im Wachstum zur Folge gehabt haben. Differenzen im Wachstumsverlauf zwischen dem heutigen Albock und den früheren Grossfelchen sind deshalb weniger auf genetische Veränderungen zurückzuführen, als vielmehr zur Hauptsache auf die Eutrophierung.

Beim Albock hat sich der Jahreszuwachs im ersten Lebensjahr um die Hälfte vergrössert. Wegen der verminderten Zunahme während der folgenden Jahre werden die Unterschiede mit steigendem Alter kleiner. Nach zwei Jahren ist der heutige Albock mit $27 \mathrm{~cm}$ Länge $6 \mathrm{~cm}$ grösser als 1944, nach vier Jahren noch $0,5 \mathrm{~cm}$ (Abb. 12).

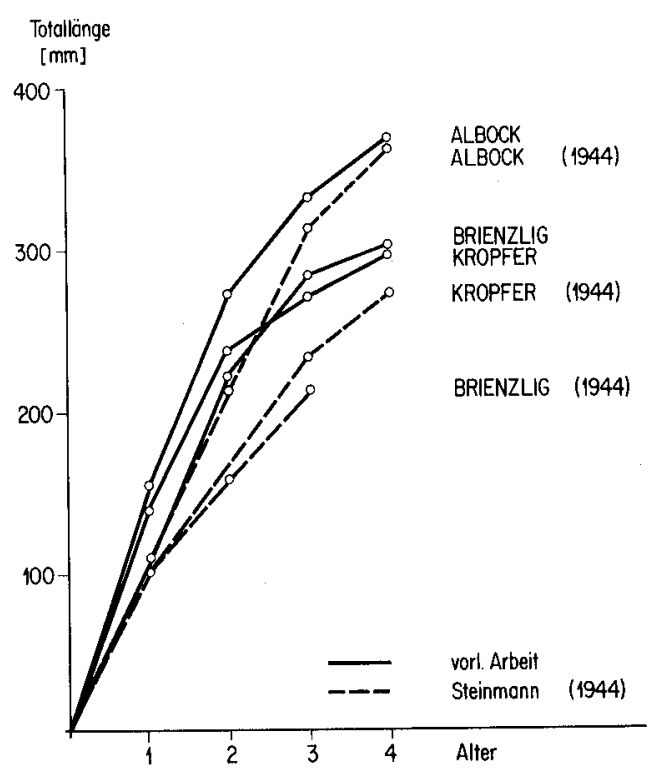

Abb. 12. Thunerseefelchen. Vergleich der heutigen Wachstumskurven mit jenen des Jahres 1944 (nach Steinmann [56]).

Figure 12. Comparison of the present growth curves of Coregonus spp. in Lake Thun with those observed by Steinmann [56] in 1944. 
Betrachtet man die früheren Längenunterschiede zwischen Brienzlig und Grossfelchen, ergibt sich nach drei Jahren eine Differenz von $10 \mathrm{~cm}$, nämlich $20-22 \mathrm{~cm}$ zu $30-32 \mathrm{~cm}$. Heute beträgt der Unterschied nur noch $5 \mathrm{~cm}$, bei Totallängen von $28 \mathrm{~cm}$ und $33 \mathrm{~cm}$.

Wie beim Albock kommt die Verbesserung des Wachstums beim Kropfer durch den grösseren Zuwachs in den ersten Jahren zustande. Der Zuwachs der späteren Jahre hat abgenommen. Der Kropfer erreicht deshalb die damalige Länge der Dreijährigen $(22-24 \mathrm{~cm})$ heute um ein Jahr früher. Nach drei Jahren ist er $4 \mathrm{~cm}$ länger als 1944 , nach vier Jahren noch $2,3 \mathrm{~cm}$.

Im Bielersee hat Roth [44] als Folge der höheren Planktonproduktion ein beschleunigtes Wachstum beim Rotauge (Rutilus rutilus) festgestellt. Wieweit ähnliche Veränderungen bei den Coregonen stattgefunden haben, ergibt ein Vergleich mit den Daten von Dottrens und Quartier [9] (Abb.13). Da bei den Felchen erste negative Einflüsse der stärkeren Verschmutzung auf das Wachstum denkbar wären, sind die Ergebnisse von besonderem Interesse.

Bei beiden Formen des Bielersees hat sich der Zuwachs im ersten Lebensjahr während der letzten 25 Jahre verdoppelt. Der Zuwachs im zweiten Lebensjahr hat sich seither nicht verändert, die Werte der folgenden Jahre haben sogar abgenommen. Für die Bondelle beträgt die Zunahme im dritten und vierten Lebensjahr heute die Hälfte, für die Palée $20-25 \%$ des früheren Wertes. Der anfängliche Längenunterschied von 8 bis $9 \mathrm{~cm}$ schrumpft deshalb bei der Bondelle bis zum vierten Altersjahr auf $1,6 \mathrm{~cm}$ zusammen. Die Palée ist bis zu diesem Zeitpunkt heute $4,2 \mathrm{~cm}$ kleiner als früher.

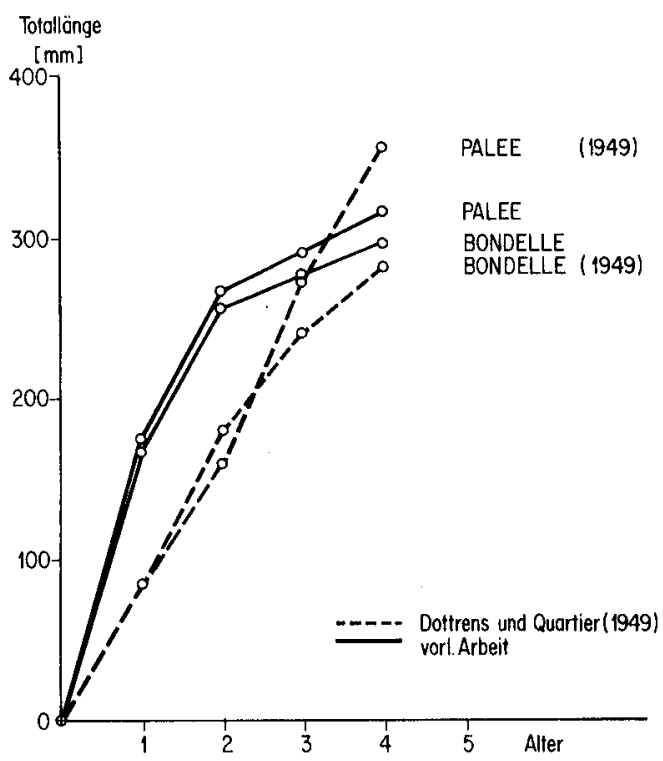

Abb. 13. Bielerseefelchen. Vergleich der heutigen Wachstumskurven mit jenen des Jahres 1949 (nach Dottrens und Quartier [9]).

Figure 13. Comparison of the present growth curves of Coregonus spp. in Lake Biel with those observed by Dottrens and Quartier [9] in 1949. 
Die ursprünglichen Längenunterschiede zwischen Bondelle und Palée nach dem dritten und vierten Lebensjahr haben sich heute auf 25-35\% der früheren Differenz reduziert (Abb.13). Zudem besitzen heute beide Formen einen ähnlichen Wachstumsverlauf, während die Kurven vor 25 Jahren beträchtlich voneinander abwichen. Es ist nicht ausgeschlossen, dass die Veränderungen durch introgressive Bastardierung (im Sinne von Mayr [31]) der beiden Formen zustande kam. Dies um so mehr, als auch im Bielersee gewisse Verlagerungen der Laichplätze festgestellt werden konnten [47]. Nach Karbe [22] ist die schwache Wachstumsverbesserung der Sandfelchen im Bodensee einerseits und die starke Wachstumserhöhung des Gangfisches andererseits (ursprünglich kleinwüchsig) auf Introgression zurückzuführen. Allerdings dürfte der Rückgang der Zuwachsraten älterer Fische kaum damit zusammenhängen (vgl. unten).

Zusammenfassend lässt sich feststellen, dass in beiden Seen und bei allen Felchenformen in den letzten Jahren eine Zunahme des Wachstums stattgefunden hat. Soweit frühere Wachstumskurven existieren, die über die ersten drei Lebensjahre hinausreichen, wird aber gleichzeitig deutlich, dass sich die Zuwachsraten älterer Fische nur wenig erhöht, sich mit steigendem Alter sogar verringert haben. Besonders auffällig ist der Rückgang des Jahreszuwachses bei den Bielerseefelchen. In beiden Seen betrifft der Rückgang hauptsächlich die schnellwüchsigen Felchenformen. Dank der massiven Erhöhung vor allem des ersten Jahreszuwachses sind die Fische trotz der erwähnten Reduktion bis nach Abschluss des Alters von drei, teilweise sogar vier und fünf Jahren heute grösser als vor 25 Jahren.

Diese Entwicklung lässt vermuten, dass den wachstumsbegünstigenden Veränderungen Faktoren entgegenwirken, die besonders mit steigendem Alter stärker in Erscheinung treten. Als wachstumsbegünstigend wirkt sich neben der Eutrophierung auch die Abnahme der Schwebstoffzufuhr aus [45]. Beide haben eine Erhöhung der Planktonproduktion zur Folge. Die Gründe für eine Wachstumshemmung sind weniger klar. Die stärkere Reduktion des Jahreszuwachses lässt an einen Zusammenhang mit der Verschmutzung denken. Jedenfalls bringt die erhöhte Planktonproduktion auch eine Zunahme der Wasserbakterien mit sich. Darunter können sich Erreger von Fischkrankheiten befinden. Wie schon Roth [44] darauf hinwies, treten Infektionen von Pseudomonas fluorescens und Aeromonas punctata häufiger in verschmutztem Wasser auf. Bacterium salmonicida überlebt nur wenige Tage in sauberem Wasser, im Gegensatz zu einem längeren Leben in verschmutztem Wasser [1]. Die eigenen Untersuchungen erstreckten sich nicht auf Krankheiten und Parasitenbefall. Die starke Infektion mit Bandwürmern, insbesondere der Bielerseefelchen, ist aber auffallend. Roth [45] erwähnt dazu, dass vor allem ältere Tiere mit Triaenophorus crassus befallen sind. Diese Umstände veranlassen uns, Krankheiten oder starken Parasitenbefall als mögliche Ursache für den Rückgang des Jahreszuwachses bei älteren Fischen (vor allem im Bielersee) anzusehen. Damit liessen sich auch der unerwartet geringe Konditionsindex und Fettgehalt [47] sowie die Tatsache, dass, trotz reichlicher Planktonproduktion in der Wachstumsperiode, Bielerseefelchen häufig leere Magen besitzen, erklären. Deufel [8] konnte entsprechende negative Auswirkungen infolge starken Befalls von Bandwürmern nachweisen. Nach Nümann [40] wurden viele, besonders junge Felchen durch Cestodeninfektion gar vernichtet. 
Im Bielersee wirkt sich die Futterkonkurrenz durch die Rotaugen als zusätzlicher Stress aus. Nach den Untersuchungen von Roth [44] hat ein Teil dieser Art ihren ursprünglichen Aufenthaltsort verlassen und wird heute im Pelagial auf den Fangplätzen der Felchen gefangen. Ihre Nahrung besteht wie die der Felchen aus Crustaceenplankton. Die bisherigen Ergebnisse scheinen zwar auf gewisse Unterschiede in der Vorliebe einzelner Plankter hinzudeuten. Inwieweit während der Wachstumsphase zwischen Felchen und Rotaugen Differenzen in der Ernährung bestehen, ist noch nicht abgeklärt. Wir dürfen aber vermuten, dass diese Futterkonkurrenz in kritischen Jahren die Entwicklung der Coregonen behindert.

\section{Konsequenzen der Wachstumsveränderungen}

Die Veränderung der Wachstumsrate hat wichtige biologische Konsequenzen: Verschiebung des Zeitpunktes der Geschlechtsreife (vgl. [47]), Veränderung der Lebensdauer. Nach den Untersuchungen von Svärdson [63] wurde die Lebensdauer langsamwüchsiger Coregonen durch Umsetzen («transplantations») in einen coregonenfreien See erhöht. Der Autor schreibt dazu: 'It seems as if early aging and slow growth are correlated.'

Die Auswirkungen der Wachstumsveränderungen auf die Lebensdauer sind an unserem Material schwierig festzustellen, da man durch die starke Befischung keine genauen Angaben über die Lebensdauer der Fische erhält. Dennoch scheinen sich die Erfahrungen von Svärdson zu bestätigen. Steinmann konnte nämlich trotz intensiven Bemühungen keine Brienzlige fangen, die älter als drei Jahre waren. Die vorliegenden Untersuchungen ergaben jedoch Tiere im siebten Altersjahr.

Die Zusammenhänge zwischen der Grösse laichender Fische, der Grösse des Eies und der Brut [7, 34] weisen darüber hinaus auf Beziehungen zwischen dem Wachstum und den Überlebenschancen der Nachkommen hin.

Vergleich des Wachstums der Thuner- und Bielerseefelchen mit Coregonen anderer Seen

Brienzlig und Kropfer des Thunersees, aber auch Palée und Bondelle des Bielersees, weisen im Gegensatz zum schnellerwachsenden Albock eine Wachstumskurve auf, die etwa jener der Blaufelchen vor der Eutrophierung entspricht [12]. Damit wachsen sie besser als der Gangfisch des Bodensees [71], besser oder ebensogut wie gewisse skandinavische [30, 59-61], englische [3], kanadische [23] und amerikanische Coregonen [6]. Erwartungsgemäss werden sie aber von der Bondella des Lago Maggiore übertroffen [4]. Deren Grössendifferenz zur Bondelle des Bielersees beträgt nach drei Jahren $2 \mathrm{~cm}$. Der Brienzlig erreicht allerdings als Einjähriger $\left(1^{+}\right)$ praktisch die monatlichen Zuwachswerte der Bondella, obwohl in bezug auf die Seetemperatur und Nahrungsmenge im Lago Maggiore die besseren Wachstumsbedingungen herrschen.

Der Abwachs des Albocks ist mit dem des Blaufelchens im Bodensee nach der Eutrophierung [38] und im Schluchsee [28] zu vergleichen. Bis zu einem Alter von drei Jahren besitzt er auch einen besseren Zuwachs als der Sandfelchen des Bodensees [71]. Übertroffen wird er von den Felchen des Starnberger Sees [25] und dem 
Lavarello des Lago Maggiore. Der Längenunterschied beträgt nach drei Jahren $4 \mathrm{~cm}$ bzw. $6 \mathrm{~cm}$. Der monatliche Zuwachs beim Albock während der Zeit des stärksten Wachstums entspricht im zweiten und dritten Altersjahr jenem des Lavarello.

\section{Jährliche Schwankungen}

Die unterschiedlichen Zuwachswerte der einzelnen Jahre sind vermutlich auf bedeutende jährliche Schwankungen des Nahrungsangebots während der Wachstumsperiode zurückzuführen. Als Ursache dafür kommen äussere Faktoren wie Abschwemmung des Planktons, Schwebstoffgehalt $[32,40]$ und Temperatur des Wassers in Frage. Ebenso wird das für die einzelnen Tiere vorhandene Nahrungsangebot durch die Grösse des Fischbestandes beeinflusst. Da wir darüber keine Angaben besitzen, bleibt das Ausmass der äusseren Faktoren auf das Nahrungsangebot unbekannt. Dass sie jedoch wesentlich dazu beitragen, lässt sich aus den mehrheitlich übereinstimmenden Wachstumsveränderungen der einzelnen Jahre im Thuner- und Bielersee vermuten. Danach verzeichneten die Felchen beider Seen im Jahre 1971 den besten Zuwachs, gefolgt vom Jahr 1969 mit unterschiedlichen Wachstumsbedingungen in den beiden Seen und den schlechten Jahren 1970 und 1968 (nur Albock). Ein Vergleich mit den Durchschnittstemperaturen und mittleren Abflussmengen der beiden Seen (Planktonabschwemmung) während der Wachstumsperiode ist deshalb von Interesse (Tab.4).

Tabelle 4. Mittlere Abflussmengen und Temperaturen während der Wachstumszeit von Mai bis September.

Table 4. Average amount of discharge and mean temperature of the lakes during the period of Coregonus growth from May to September.

\begin{tabular}{|c|c|c|c|c|}
\hline Jahr & $\begin{array}{l}\text { Thunersee } \\
\text { Abflussmengen } \\
\text { (Aare, Thun) } \\
\left(\mathrm{m}^{3} / \mathrm{sec}\right)\end{array}$ & $\begin{array}{l}\text { Temperatur } \\
\text { (Aare, Interlaken) } \\
\left({ }^{\circ} \mathrm{C}\right)\end{array}$ & $\begin{array}{l}\text { Bielersee } \\
\text { Abflussmengen } \\
\text { (Aare, Brügg) } \\
\left(\mathrm{m}^{3} / \mathrm{sec}\right) \\
\end{array}$ & $\begin{array}{r}\text { Temperatur } \\
\text { (Aare, Brügg) } \\
\left({ }^{\circ} \mathrm{C}\right) \\
\end{array}$ \\
\hline 1971 & 144 & 13,6 & 216 & 16,6 \\
\hline 1970 & 225 & 12,1 & 403 & 14,7 \\
\hline 1969 & 177 & 13,0 & 319 & 15,5 \\
\hline 1968 & 173 & 13,1 & 346 & 15,3 \\
\hline
\end{tabular}

(Nach Angaben des Eidg. Amtes für Energiewirtschaft)

Aus der Tabelle geht hervor, dass in beiden Seen, gemäss der Stärke der entsprechenden Zuwachsrate der Felchen, im Jahre 1971 wesentlich günstigere Bedingungen (Abflussmengen und Temperatur) als in den übrigen Jahren herrschten. Für den Bielersee kommt auch zum Ausdruck, dass im Jahre 1969 gegenüber 1970 und 1968 bessere Voraussetzungen für den Planktonbestand existierten. Wie nach der Grösse des Zuwachses liegt das Jahr 1969 also auch bezüglich mittlerer Temperatur und Abflussmengen an dritter Stelle.

Im Thunersee ist aus der Tabelle einzig für das Jahr 1969 kein Zusammenhang mit den besseren Zuwachswerten gegenüber 1968 beim Albock ersichtlich. Im allgemeinen kommen aber die durch die Zuwachswerte gekennzeichneten Wachstumsbedin- 
gungen in den einzelnen Jahren, wenn auch weniger deutlich, ebenfalls zum Ausdruck.

Wir können daraus schliessen, dass die Witterung via Wassertemperatur und Abflussmengen besonders im Bielersee einen massgebenden Einfluss auf das Nahrungsangebot ausübt und daher bestimmte Jahre einen besseren Zuwachs erlauben als andere (vgl. [4, 12, 37].

\section{Zusammenfassung}

Die Ergebnisse der vorliegenden Arbeit sollen als Basis zu einer zweckmässigen Bewirtschaftung der Coregonenpopulationen im Thuner- und Bielersee dienen. Die Untersuchungen wurden besonders aktuell, als sich Änderungen in der arten- und rassenmässigen Zusammensetzung sowie der Biologie dieser Coregonen als Folge der Eutrophierung feststellen liessen.

Aufgrund der Magenanalysen ist für die Ernährung der Thuner- und Bielerseefelchen während der jährlichen Wachstumsperiode (Mai bis August, September) der hohe Anteil an Cladoceren charakteristisch (Abb. 1-4). Im Winter findet man in den Magen der Felchen vermehrt Copepoden und Bodenorganismen, hauptsächlich Chironomiden. Als bevorzugte Planktonorganismen von Albock und Brienzlig gelten Daphnia, Bythotrephes und Leptodora (beim Brienzlig auch Cyclops); Daphnia und Leptodora, schwächer Bosmina und Cyclops von den Bielerseefelchen. Mit Ausnahme einer kurzen Zeitspanne im Zusammenhang mit der Laichzeit sind Differenzen in der Ernährung eher auf Grössenunterschiede als auf verschiedene Ernährung der Populationen zurückzuführen. Während der Bondelle-Laichzeit frisst die Mehrheit der unreifen und verlaichten Palées ausschliesslich BondelleEier, während sich die Bondelle von Daphnia ernährt. Zwischen Brienzlig und Albock differiert die Zusammensetzung der Nahrung wenig oder überhaupt nicht. Einzig jene des Kropfers (Bodenorganismen: Chironomiden, kleine Eier) scheint sich während des ganzen Jahres von den übrigen Formen zu unterscheiden.

Die Wachstumscharakteristiken wurden anhand von Altersbestimmungen an Schuppen, nach Berechnung der Beziehung Schuppenlänge zu Fischlänge und unter Benützung des Segerstraleschen Korrekturverfahrens, bestimmt (Abb. 7, 8). Als mittlere asymptotische Länge $\mathrm{L}_{\infty}$ und Fords Wachstumskoeffizient $\mathrm{k}$ ergeben sich für den Albock $\mathrm{L}_{\infty}=44 \mathrm{~cm}, \mathrm{k}=0,58$, für den Brienzlig $\mathrm{L}_{\infty}=36 \mathrm{~cm}, \mathrm{k}=0,55$ und für die Bondelle $\mathrm{L}_{\infty}=31 \mathrm{~cm}, \mathrm{k}=0,43$. Das Wachstum der Thuner- und Bielerseefelchen hat sich in den letzten 25 Jahren stark verbessert und entspricht heute etwa demjenigen der Bodenseeblaufelchen vor der Eutrophierung (Albock: Schluchseefelchen oder Bodenseeblaufelchen nach der Eutrophierung). Der Brienzlig beispielsweise ist heute nach drei Jahren mit $28 \mathrm{~cm}$ Länge durchschnittlich $7 \mathrm{~cm}$ länger. Im Vergleich zum Albock (Grossfelchen) mit $33 \mathrm{~cm}$ Länge besteht nur noch ein Unterschied von $5 \mathrm{~cm}$ gegenüber von $10 \mathrm{~cm}$ Anno 1944 (Abb. 12). Die Längenzunahme ist vor allem auf eine Verbesserung des Zuwachses während der ersten beiden Lebensjahre zurückzuführen. So hat sich bei den Bielerseefelchen die Länge der Fische nach einem Lebensjahr verdoppelt (Abb. 13). Später liegen die Zuwachswerte unter den früheren. Der Rückgang ist, vor allem bei den Bielerseefelchen, unter anderem vermutlich auf Krankheiten und Parasitenbefall zurückzuführen. 
Die Wachstumsperiode erstreckt sich von Mai bis September, Oktober, mit durchschnittlichem Zuwachs pro Monat während des stärksten Wachstums von 1,4, 0,9 und $0,3 \mathrm{~cm}$ bei Albock, Brienzlig bzw. bei der Bondelle (zweites Altersjahr) (Abb.9-11). Die Werte des Albocks sind vergleichbar mit jenen des Lavarello des Lago Maggiore.

Als Folge des Nahrungsangebots, welches seinerseits von der Witterung via Wassertemperatur und Abflussmengen beeinflusst wird, variiert der jährliche Zuwachs der Felchen. Nach dessen Stärke lassen sich die einzelnen Jahre folgendermassen einordnen:

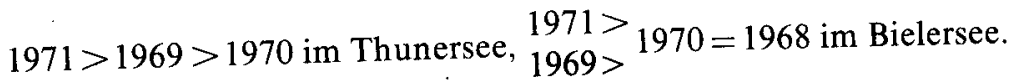

\section{SUMMARY}

Nutrition and growth of the coregonid fish populations in Lake Thun and Lake Biel

The results of the present investigation shall serve as a basis to a purposeful management of the Coregonus spp. of Lake Thun and Lake Biel. This paper became necessary, when changes in the biology of the fish and in the composition of stocks, as a consequence of eutrophication, were observed.

According to stomach analyses, Cladocera are the main source of food of the Coregonus spp. populations in Lake Thun (local names: Kropfer, Albock, Brienzlig) and Lake Biel (local names: Palée, Bondelle). In winter and spring they feed more on Copepods and bottom organisms, mainly Chironomus (fig. 1-4). Daphnia, Bythotrephes and Leptodora are the favourite plankton organisms of Albock and Brienzlig (Brienzlig also Cyclops); Daphnia and Leptodora, less Bosmina and Cyclops, are those of the stocks in Lake Biel (proportion in stomach higher than in lake plankton). Except for a short period around the spawning season, differences in feeding habits are attributable rather to fish size than to population type. During the Bondelle spawning period the majority of the premature and spawned Palée prey on the eggs of the Bondelle, while the latter feed on Daphnia. The composition of the food of Brienzlig and Albock differs little or not at all. The feeding habits of the Kropfer however, which concentrates mainly on bottom organisms (Chironomus, little eggs), probably differ from those of other species during the entire year.

Growth characteristics were determined on the basis of age determinations through scales calculated according to the relation between scale length and total fish length and subject to curve adjustment as illustrated by the Segerstrale correction procedure (fig. 7,8 ). The following mean asymptotic length $\mathbf{L}_{\infty}$ and Ford's growth coefficient $\mathrm{k}$ were calculated: Albock: $\mathrm{L}_{\infty}=44 \mathrm{~cm}, \mathrm{k}=0.58$; Brienzlig: $\mathrm{L}_{\infty}=36 \mathrm{~cm}$, $\mathrm{k}=0.55$; Bondelle: $\mathrm{L}_{\infty}=31 \mathrm{~cm}, \mathrm{k}=0.43$. In the past 25 years the Coregonus spp. in Lake Thun and Lake Biel have experienced a considerable improvement in their growth rate, which corresponds to that of the Blaufelchen of Lake Constance prior to eutrophication (Albock corresponds to species of Schluchsee and to Blaufelchen of Lake Constance after eutrophication). At an age of three and a length of $28 \mathrm{~cm}$ the present Brienzlig is $7 \mathrm{~cm}$ longer as it used to be. Compared to the Albock (Grossfelchen), the difference is only $5 \mathrm{~cm}$ versus $10 \mathrm{~cm}$ in 1944 (fig. 12). This improvement is mainly referred to better growth rates during the first two years: those of Palée and Bondelle have doubled (fig. 13). Later growth rates lie below former values. The decrease is probably caused, among others, by diseases and parasites.

Growth is limited to the period between May and September/October, with an average monthly increment during the period of best growth of 1.4, 0.9 and $0.3 \mathrm{~cm}$ for Albock, Brienzlig and Bondelle, respectively (age two) (fig.9-11). The values of the Albock can be compared to those of the Lavarello of the Lago Maggiore.

Due to food supply, which is influenced by the weather via water temperature and the amount of lake discharge, the annual increase of th Coregonus ssp. varies. According to their magnitude the years can be arranged as follows: $1971>1969>1970$ Lake Thun,

$1971>1970>1968$ Lake Biel (tables 1-3).

$1969>$ 


\section{RÉSUMÉ}

Nutrition et croissance des populations de Corégones (Coregonus ssp.) dans les lacs de Thoune et de Bienne Les résultats des présentes recherches doivent servir de base à un aménagement rationnel des Corégones des lacs de Thoune et de Bienne. Ce travail est devenu actuel lorsque des changements dans les espèces et rasses ainsi que dans la biologie des Corégones se sont produits à la suite de l'eutrophisation.

Selon les analyses du contenu stomacal, ce sont les Cladocères qui, pendant la période annuelle de croissance (mai-août, septembre), fournissent la plus grande partie de la nourriture des Corégones du lac de Thoune (noms locaux: Kropfer, Albock, Brienzlig) et des Corégones du lac de Bienne (noms locaux: Palée, Bondelle) (fig. 1-4). En hiver et au printemps, ils se nourrissent davantage de Copépodes et d'organismes benthiques, surtout des Chironomides. Les organismes planctoniques préférés des Albock et des Brienzlig sont Daphnia, Bythotrephes et Leptodora (chez le Brienzlig aussi Cyclops); Daphnia et Leptodora, moins fréquemment Bosmina et Cyclops, sont les favorits des Corégones du lac de Bienne (la proportion dans l'estomac est plus élevée que dans le plancton du lac). Excepté pour une courte période, correspondant à la période de frai, les différences dans l'alimentation doivent être attribuées davantage aux différentes tailles des poissons qu'au type de population. Pendant la période de reproduction des Bondelles, la plupart des Yalées immatures et celles qui ont déjà frayé mangent exclusivement des œufs de Bondelle, tandis que les Bondelles-mêmes se nourrissent de Daphnia. Il n'y a que très peu ou pas de différence entre la composition de la nourriture des Brienzlig et celle des Albock. Cependant la nourriture des Kropfer se compose essentiellement d'organismes benthiques (Chironomides et petits oufs) et diffère probablement des autres espèces pendant toute l'année.

Les caractéristiques de croissance ont été déterminées sur la base de la détermination d'âge à l'aide des écailles et calculées d'après la relation entre la longueur de l'écaille et la longueur totale du poisson en ajustant la courbe selon la méthode de correction de Segerstrale (fig.7, 8). On a calculé la longueur asymptotique moyenne $\mathrm{L}_{\infty}$ et le coefficient de croissance de Ford $\mathrm{k}$ : Albock: $\mathrm{L}_{\infty}=44 \mathrm{~cm}, \mathrm{k}=0,58$; Brienzlig: $\mathrm{L}_{\infty}=36 \mathrm{~cm}, \mathrm{k}=0,55$; Bondelle: $\mathrm{L}_{\infty}=31 \mathrm{~cm}, \mathrm{k}=0,43$. La croissance des Corégones des lacs de Thoune et de Bienne s'est fortement améliorée ces 25 dernières années et atteint aujourd'hui celle des Blaufelchen du lac de Constance avant l'eutrophisation (celle de l'Albock correspond aux espèces du lac de Schluchsee et aux Blaufelchen du lac de Constance après l'eutrophisation). Aujourd'hui, par exemple, un Brienzlig âgé de trois ans a une longueur de $28 \mathrm{~cm}$, c'est-à-dire en moyenne $7 \mathrm{~cm}$ de plus qu'autrefois. En comparaison avec l'Albock (Grossfelchen) long de $33 \mathrm{~cm}$, la différence n'est plus que de $5 \mathrm{~cm}$, contre $10 \mathrm{~cm}$ en 1944 (fig. 12). L'augmentation de la longueur est dûe avant tout à une amélioration de la croissance pendant les deux premières années. Ainsi les longueurs des Palées et des Bondelles ont doublés pendant une seule année (fig. 13). Plus tard, les valeurs d'accroissement restent en dessous des précédentes. Cette diminution est dûe entre autre à des maladies et parasites.

La croissance est limitée à la période comprise entre mai et septembre/octobre, avec une augmentation mensuelle moyenne pendant la croissance la plus forte de 1,4 (Albock), 0,9 (Brienzlig) et 0,3 (Bondelle), (deuxième année) (fig.9-11). Les valeurs de l'Albock peuvent être comparées à celles du Lavaret dans le lac Majeur.

La variation de l'augmentation annuelle des Corégones est dûe à l'offre de nourriture, laquelle est influencée par les conditions météorologiques, indirectement par la température de l'eau et l'écoulement. Selon l'ampleur de cette variation, on peut arranger les années dans l'ordre suivant:

$1971>1969>1970$ dans le lac de Thoune, $1971>1969>1970>1968$ dans le lac de Bienne (tabl.1-3).

\section{VERDANKUNG}

Ich danke den Herren Prof. Dr. W. Stumm, Direktor der EAWAG, und Herrn Prof. Dr. O. Jaag, alt Direktor, dass sie die Durchführung der Arbeit ermöglichten. Besonderer Dank gebührt dem Leiter der Abteilung Fischereiwissenschaften an der EAWAG, Herrn Dr. W. Geiger, der die Arbeit anregte und mit seiner Hilfe unterstützte. Mein herzlicher Dank gilt ferner Prof. Dr. A. Berg vom EURATOM in Ispra für die Durchführung eines Teils der Wachstumsberechnungen und wertvolle Diskussionen, Herrn Prof. Dr. E. Grimaldi vom Istituto Italiano di Idrobiologia in Pallanza für anregende Diskussionen, Herrn Prof. Dr. H. Roth, Kantonaler Fischereiinspektor des Kantons Bern, für stetiges Interesse und persönliche Unterstützung, den Fischereiaufsehern, Herren W. Röthlisberger, H. Fahrni, H. Greber, 
H. Roth, A. Wittwer, H. Perrod, H. Ballif, und den Berufsfischern des Brienzer-, Thuner- und Bielersees, vor allem den Herren H. Kaufmann, R. und F. Thomann, A. und E. Meyer, W. Sieber, H. Tschanz, H. Tschan, K. Furer, A. Martin, H. Pilloud, A. Grimm, H. Dubler und H.P. Dasen, für ihre stete Hilfe bei den vielen Probenahmen. Schliesslich danke ich Herrn Dr. A. Keller für die Durchsicht des Manuskripts.

Die Arbeit wurde mit Unterstützung der «Stiftung der Wirtschaft zur Förderung des Gewässerschutzes in der Schweiz» durchgeführt.

\section{LITERATURVERZEICHNIS}

1 Amlacher, E.: Taschenbuch der Fischkrankheiten, 286 S. Gustav Fischer, Jena 1961.

2 Auerbach, M., Maerker, W., und Schmalz, J.: Hydrographisch-biologische Bodensee-Untersuchungen. I. Ergebnisse der Jahre 1920-1922. Arch. Hydrobiol., Suppl. 3, 597-738 (1924).

3 Bagenal, T.B: Notes on the biology of schelly Coregonus lavaretus (L.) in Haweswater and Ullswater. J. Fish Biol. 2, 137-154 (1970).

4 Berg, A., und Grimaldi, E.: Biologia delle due forme di Coregone (Coregonus sp.) del Lago Maggiore. Memorie Ist. ital. Idrobiol. 18, 25-196 (1965).

5 Berg, A., und Grimaldi, E.: A critical interpretation of the scale structures used for the determination of annuli in fish growth studies. Memorie Ist. ital. Idrobiol. 21, 225-239 (1967).

6 Brown, E. H.: Extreme female predominance in the bloater (Coregonus hoyi) of lake Michigan in the 1960's. In: Lindsey, C.C., und Woods, C.S. (Hrsg.): Biology of Coregonid fishes, 560 S. University of Manitoba Press, Winnipeg 1970.

7 Brown, M.E.: Experimental studies on growth. In: Brown, E.M. (Hrsg.): The physiology of fishes, Vol. 1. Academic Press Inc., Publishers, New York 1957.

8 Deufel, J.: Auswirkungen des Cestodenbefalls bei Blaufelchen. Arch. FischWiss. 7, 145-150 (1956).

9 Dottrens, E., und Quartier, A.: Les Corégones du Lac de Neuchâtel. Etude biométrique. Revue suisse Zool. 56, 689-730 (1949).

10 Einsele, W.: Über das Wachstum der Coregonen im Voralpengebiet, insbesondere über das Verhältnis von Schuppen- und Längenwachstum. Z. Fisch. Hilfswiss. 41, 23-45 (1943).

11 Elster, H.J.: Beiträge zur Biologie des Blaufelchen (Coregonus wartmanni Bloch). Int. Rev. Hydrobiol. Hydrogr. 30, 181-246 (1934).

12 Elster, H.J.: Über das Verhältnis von Produktion, Bestand, Befischung und Ertrag sowie über die Möglichkeiten einer Steigerung der Erträge, untersucht am Beispiel der Blaufelchenfischerei des Bodensees. Z. Fisch. Hilfswiss. 62, 169-357 (1944).

13 Elster, H.J.: Probleme der Felchenaufzucht im Bodensee. Allg. FischZtg. 75, 58-60 (1950).

14 Fabricius E., und Lindroth, A.: Experimental observations of the spawning of whitefish, Coregonus lavaretus $\mathrm{L}$., in the stream aquarium of the Hölle Laboratory at River Indalsälven. Rep. Inst. Freshwat. Res. Drottningholm 35, 105-112 (1954).

15 Fatio, V.: Faune des Vertébrés de la Suisse, Vol. V, 2e partie. Genf und Basel 1890.

16 Florin, J.: Berichte über die Ergebnisse von Untersuchungen an Blaufelchen-Sonderfängen aus dem Bodensee. Mitt. Kant. Amts Gewässerschutz St. Gallen (1970-1973).

17 Freidenfelt, T.: Untersuchungen über die Coregonen des Wenersees. Int. Rev. Hydrobiol. Hydrogr. $30,49-163$ (1934).

18 Galbraith, J.: Size selective predation on Daphnia by rainbow trout and yellow perch. Trans. Am. Fish. Soc. 96, 1-10 (1967).

19 Hagen, H.K.: Age, growth and reproduction of the mountain whitefish in Phelps Lake, Wyoming. In: Lindsey, C.C., und Woods, C.S. (Hrsg.): Biology of Coregonid fishes, 560 S. University of Manitoba Press, Winnipeg 1970.

20 Haram, O.J., und Jones, J. W.: Some observations on the food of the gwyniad Coregonus clupeoides pennantii Valenciennes of Llyn Tegid (Lake Bala), North Wales. J. Fish Biol, 3, 287-295 (1971).

21 Hogman, W.J.: Early scale development on the Great Lakes Coregonids, Coregonus artedii and C. kiyi. In: Lindsey, C.C., und Woods, C.S. (Hrsg.): Biology of Coregonid fishes, 560 S. University of Manitoba Press, Winnipeg 1970.

22 Karbe, L.: Die Auswirkung der künstlichen Eutrophierung des Bodensees auf das Artgefüge seiner Coregonenpopulationen. Mitt. hamb. zool. Mus. Inst. (Kosswig-Festschr., S.83-90 (1964). 
23 Kennedy, W.A.: Tagging returns, age studies and fluctuations in abundance of Lake Winnipeg Whitefish, 1931-1951. J. Fish. Res. Bd Canada 11, 284-309 (1954).

24 Kliever, E.V.: Gillraker variation and diet in lake whitefish Coregonus clupeaformis in Northern Manitoba. In: Lindsey, C.C., und Woods, C.S. (Hrsg.): Biology of Coregonid fishes, $560 \mathrm{~S}$. University of Manitoba Press, Winnipeg 1970.

25 Koelbing, A.: Der Starnberger See und die seinem Trophiezustand angemessene Bewirtschaftungsweise des Coregonenbestandes. Veröff. zool. StSamml., Münch. 17, 1-108 (1974).

26 Koschin, N.I.: Die Nahrung der Maränen (Coregonus lavaretus lavaretoides (Pol.) Berg) in der Schala-Bucht des Onega-Sees. Rep. Karelia Sci. Fish. Stat. 1, 359-373 (1935).

27 Krinner, A.: Mageninhaltsuntersuchungen an Fischen aus dem Kochel- und Walchensee. Arch. Hydrobiol. 14, 97-115 (1924).

28 Lampert, W.: Untersuchungen zur Biologie und Populationsdynamik der Coregonen im Schluchsee. Arch. Hydrobiol., Suppl. 38, 237-314 (1971).

29 Lee, R.M.: An investigation into the methods of growth determination in fishes. Cons. Explor. Mer, Publ. Circonstance 63, $35 \mathrm{~S}$. (1912).

30 Lindstrøm, T.: On the importance of growth and spawning site ecology of whitefish (Coregonus) for the survival of the young. Rep. Inst. Freshwat. Res. Drottningholm 47, 128-146 (1967).

31 Mayr, E.: Artbegriff und Evolution, 617 S. Paul-Parey-Verlag, Hamburg und Berlin 1967.

32 Minder, L.: Untersuchungen am Bielersee. Vjahrsschr. natf. Ges. Zürich 8l, 107-176 (1936).

33 Mittelholzer, E.: Populationsdynamik und Produktion des Zooplanktons im Greifensee und im Vierwaldstättersee. Schweiz. Z. Hydrol. 32, 90-149 (1969).

34 Nikolskii, G.V.: Die Selbstregulation der Populationsstärke bei Fischen. Sowjetwiss., naturw. Beitr. 2, 113-125 (1961).

35 Nilsson, N. A.: On the food competition between two species of Coregonus in a North Swedish lake. Rep. Inst. Freshwat. Res. Drottningholm 39, 146-161 (1958).

36 Nümann, W.: Vorläufiger Bericht über das stark veränderte Wachstum der Blaufelchen im Bodensee und Versuch einer Fangprognose für das Jahr 1958. Allg. FischZtg 83, 88-91 (1958).

37 Nümann, W.: Das Wachstum der Blaufelchen und seine Berechnung bei Erfassung aller Individuen der einzelnen Jahrgänge in Abhängigkeit von der Stärke des Jahrganges, den jährlich variierenden Aussenfaktoren, der Eutrophierung und den regionalen Unterschieden des Sees. Arch. FischWiss. 10, 5-20 (1959).

38 Nümann, W.: Die Eutrophierung des Bodensees, die Zunahme der Barsche und die intensive Befischung als Ursachen für den rapiden Rückgang der Blaufelchenfänge. Allg. FischZtg 89, 308311 (1964).

39 Nümann, W.: Versuch einer Begründung für den Wandel in der qualitativen und quantitativen Zusammensetzung des Fischbestandes im Bodensee während der letzten 60 Jahre und eine Bewertung der Besatzmassnahmen. Schweiz. Z. Hydrol. 35, 206-238 (1974).

40 Nydegger, P.: Vergleichende limnologische Untersuchungen an sieben Schweizerseen. Beitr. Geol. Schweiz, Hydrol. 9, 1-80 (1957).

41 Olofsson, O.: Nagra inplanteringar av Lomsjö-sik. Svensk FiskTidskr. 43, 4-8, 43-47, 74-79 (1934).

42 Pirocchi, L.: Struttura e vicenda delle biocenosi mesoplanctiche del Lago Maggiore. Memorie Ist. ital. Idrobiol. 3, 59-119 (1947).

43 Ricker, W.E.: Computation and interpretation of biological statistics of fish populations. Bull. Fish. Res. Bd Canada 191, 382 S. (1975).

44 Roth, H.: Das Weissfischproblem in der Schweiz. Veröff. Eidg. Amtes Gewässerschutz Eidg. Fischereiinspektion 26, 69 S. (1969).

45 Roth, H.: Fischereibiologische Probleme in Brienzer-, Thuner- und Bielersee. Veröff. Eidg. Amtes Gewässerschutz Eidg. Fischereiinsp. 29, 45-79 (1972).

46 Roth, H., und Geiger, W.: Brienzersee, Thunersee, and Bielersee: effects of exploitation and eutrophication on the salmonid communities. J. Fish. Res. Bd Canada 29, 755-764 (1972).

47 Rufli, H.: Die Biologie der Coregonen des Thuner- und Bielersees. Diss. Nr.5451 EAWAG/ETH Zürich (1975).

48 Rufli, H.: Die heutigen sympatrischen Felchenpopulationen (Coregonus spp.) des Thuner- und Bielersees und ihre Morphologie. Schweiz. Z. Hydrol. 40/1, 7-31 (1978).

49 Runnström, S.: Om smärlingen fran nagra Jämtlandssjöar. Svensk FiskTidskr. 50, 25-29 (1944). 
50 Schröder, R.: Untersuchungen über die Planktonverteilung mit Hilfe der Unterwasser-Fernsehanlage und des Echographen. Arch. Hydrobiol., Suppl. 25, 228-241 (1961).

51 Schröder, R., und Schröder, H.: On the use of the echo sounder in lake investigations. Memorie Ist. ital. Idrobiol. 17, 81-103 (1964).

52 Segerstrale, C.: Über scalimetrische Methoden zur Bestimmung des linearen Wachstums bei Fischen, insbesondere bei Leuciscus idus L., Abramis brama L. und Perca fluviatilis L. Acta zool. fenn. 15, 1-169 (1933).

53 Siebeck, O.: Untersuchungen über die Vertikalwanderung planktischer Crustaceen unter Berücksichtigung der Strahlungsverhältnisse. Int. Rev. Hydrobiol. 45 (1960).

54 Slack, H.D., Gervers, F.W.K., und Hamilton, J.D.: The biology of the powan. Glasg. Univ. Publ. Stud. Loch Lomond 1, 113-127 (1957).

55 Southern, R., und Gardiner, A.C.: The seasonal distribution of the plankton in Lough Derg and the River Shannon. Scient. Invest. Fish. Brch Ire. 1.(1926).

56 Steinmann,P.: Gutachten über die Fischereiverhältnisse des Thunersees. Kant. Forstdir. Kt. Bern, 22. Juli 1944 (1944).

57 Steinmann, P.: Monographie der Schweizerischen Koregonen. Schweiz. Z. Hydrol. 12/1-2, 13/1 (1950/51).

58 Svärdson, G.: The Coregonid problem. I. Some general aspects of the problem. Rep. Inst. Freshwat. Res. Drottningholm 29,89-101 (1949).

59 Svärdson, G.: The Coregonid problem. II. Morphology of two Coregonid species in different environments. Rep. Inst. Freshwat. Res. Drottningholm 31, 79-125 (1950).

60 Svärdson, G.: The Coregonid problem. III. Whitefish from the Baltic, successfully introduced into fresh waters in the north of Sweden. Rep. Inst. Freshwat. Res. Drottningholm 32, 79-125 (1951).

61 Svärdson, G.: The Coregonid problem. V. Sympatric whitefish species of the lakes Idsjön, Storsjön and Hornavan. Rep. Inst. Freshwat. Res. Drottningholm 34, 141-166 (1953).

62 Svärdson, G.: The Coregonid problem. VII. The isolating mechanisms in sympatric species. Rep. Inst. Freshwat. Res. Drottningholm 46, 95-123 (1965).

63 Svärdson, G.: Significance of introgression in Coregonid evolution. In: Lindsey, C.C., und Woods, C.S. (Hrsg.): Biology of Coregonid fishes, 560 S. University of Manitoba Press, Winnipeg 1970.

64 Swynnerton, G.H., und Worthington, E. B.: Note on the food of fish in Haweswater (Westmorland). J. Anim. Ecol. 9, 183-187 (1940).

65 Thienemann, A.: Über den Coregonen des Comer Sees. Arch. Hydrobiol. 19, 666-668 (1928).

66 Tonolli, V.: Ricerche sulla microstruttura di distribuzione dello zooplancton nel Lago Maggiore. Memorie Ist. ital. Idrobiol. 10, 125-152 (1958).

67 Valtonen, T.: The selected temperature of Coregonus nasus (Pallas) sensu Svärdson, in natural waters compared with some other fish. In: Lindsey, C.C., und Woods, C.S. (Hrsg.): Biology of Coregonid fishes, $560 \mathrm{~S}$. University of Manitoba Press, Winnipeg 1970.

68 Wagler, E.: Die Coregonen in den Seen des Voralpengebietes. VIII. Über die Eier und Brut der Bodenseecoregonen. Arch. Hydrobiol. 25, 1-21 (1933).

69 Wagler, E.: Die Coregonen in den Seen des Voralpengebietes. VII. Der Kilch des Bodensees (Coregonus acronius von Rapp.). Int. Rev. Hydrobiol. 30, 1-48 (1934).

70 Wagler, E.: Die Coregonen in den Seen des Voralpengebietes. IX. Die Systematik der Voralpencoregonen. Int. Rev. Hydrobiol. 35, 345-446 (1937).

71 Wagler, E.: Die Lachsartigen. II. Teil: Coregonen. Handb. Binnenfisch. Mitteleur. 3, 369-501 (1941).

72 Wells, L., und Beeton, A.: Food of the bloater, Coregonus hoyi, in lake Michigan. Trans. Am. Fish. Soc. 92/3, 245-255 (1963).

Adresse des Autors: Dr. Hans Rufli, Seenforschungslaboratorium der EAWAG/ETH, Abt. Fischereiwissenschaften, CH-6047 Kastanienbaum. 